\title{
Largest temperature of the radiation era and its cosmological implications
}

\author{
Gian Francesco Giudice* \\ CERN Theory Division, CH-1211 Geneva 23, Switzerland
}

Edward W. Kolb

NASA/Fermilab Astrophysics Center, Fermi National Accelerator Laboratory, Batavia, Illinois 60510-0500 and Department of Astronomy and Astrophysics, Enrico Fermi Institute, The University of Chicago, Chicago, Illinois 60637-1433

\author{
Antonio Riotto \\ Scuola Normale Superiore, Piazza dei Cavalieri 7, I-56126 Pisa, Italy \\ and INFN, Sezione di Pisa, I-56127 Pisa, Italy
}

(Received 15 May 2000; revised manuscript received 17 May 2001; published 13 June 2001)

\begin{abstract}
The thermal history of the universe before the epoch of nucleosynthesis is unknown. The maximum temperature in the radiation-dominated era, which we will refer to as the reheat temperature, may have been as low as $0.7 \mathrm{MeV}$. In this paper we show that a low reheat temperature has important implications for many topics in cosmology. We show that weakly interacting massive particles (WIMP's) may be produced even if the reheat temperature is much smaller than the freeze-out temperature of the WIMP, and that the dependence of the present abundance on the mass and the annihilation cross section of the WIMP differs drastically from familiar results. We reexamine predictions of the relic abundance and resulting model constraints of supersymmetric dark matter, axions, massive neutrinos, and other dark matter candidates, nucleosynthesis constraints on decaying particles, and leptogenesis by decay of superheavy particles. We find that the allowed parameter space of supersymmetric models is altered, removing the usual bounds on the mass spectrum; the cosmological bound on massive neutrinos is drastically changed, ruling out Dirac (Majorana) neutrino masses $m_{\nu}$ only in the range $33 \mathrm{keV} \lesssim m_{\nu} \lesssim 6$ (5) MeV, which is significantly smaller from the standard disallowed range $94 \mathrm{eV} \leq m_{\nu} \leq 2 \mathrm{GeV}$ (this implies that massive neutrinos may still play the role of either warm or cold dark matter); the cosmological upper bound on the Peccei-Quinn scale may be significantly increased to $10^{16}$ $\mathrm{GeV}$ from the usually cited limit of about $10^{12} \mathrm{GeV}$; and that efficient out-of-equilibrium grand unified theory GUT baryogenesis and/or leptogenesis can take place even if the reheat temperature is much smaller than the mass of the decaying superheavy particle.
\end{abstract}

DOI: 10.1103/PhysRevD.64.023508

\section{INTRODUCTION}

The initiation of the radiation-dominated era of the universe is believed to result from the decay of coherent oscillations of a scalar field whose energy dominated the universe before decay. The decay of the coherent oscillations of the scalar field and the subsequent thermalization of the decay products is known as reheating. ${ }^{1}$

The reheat process is often associated with the final stage of inflation. However, reheating could have been episodic, with several reheat events after inflation. We will be interested in the final reheating before primordial nucleosynthesis, which may just as well have been the result of the decay of a weakly coupled scalar field unrelated to inflation, for instance a modulus. For this reason the scalar field $\phi$, whose decay leads to reheating, will not be referred to as the inflaton.

\footnotetext{
*On leave of absence from INFN, Sezione di Padova, Padua, Italy.

${ }^{1}$ While we discuss reheating as the decay of coherent field oscillations, we only make use of the fact that the energy density of coherent field oscillations scales in expansion as $a^{-3}$, where $a$ is the Friedmann-Robertson-Walker scale factor. One could just as easily imagine that the universe is dominated by some unstable massive particle species, rather than coherent oscillations of a scalar field.
}

PACS number(s): $98.80 . \mathrm{Cq}$

A common assumption is that many of the interesting cosmological phenomena accessible to present-day observations occurred after reheating, during the radiationdominated phase of the early universe. This seems to be a reasonable assumption, since inflation [1] erases any initial condition on the number densities of ordinary particles, while the reheat process repopulates the universe. The assumption of an initial condition of thermal and chemical equilibrium in a radiation-dominated universe is then equivalent to the hypothesis that the maximum temperature obtained during the radiation-dominated era, $T_{R H}$, is larger than characteristic temperatures of cosmological processes under investigation.

The fact that we have no physical evidence of the radiation-dominated era before the epoch of nucleosynthesis (i.e., temperatures above about $1 \mathrm{MeV}$ ) is a simple, but crucial, point. Therefore, a priori one should consider $T_{R H}$ as an unknown quantity that can take any value as low as about 1 $\mathrm{MeV}$. Indeed, there are good physical motivations for studying cosmologies with very low $T_{R H}$.

In theories in which the weakness of gravity is explained by large compactified extra dimensions [2], emission of Kaluza-Klein gravitons in the bulk constrains the normalcy temperature at which the radius of the compactified dimensions is stabilized with vanishing energy density in the compactified space to be in the $\mathrm{MeV}$ to $\mathrm{GeV}$ region [3]. In prac- 
tice, the stabilization occurs in a post-inflationary phase, and therefore we can identify the normalcy temperature with $T_{R H}$.

Supergravity and superstring theories usually have particles, such as a gravitino or a modulus, with only gravitational interactions. Late decay of these particles may jeopardize the success of standard big-bang nucleosynthesis [4]. This problem can be solved by assuming sufficiently low $T_{R H}$, of the order of $10^{8}-10^{10} \mathrm{GeV}$ [5]. Moreover, it has been recently realized [6] that nonthermal production of these gravitational relics during the inflationary phase can impose upper bounds on $T_{R H}$ as low as $100 \mathrm{GeV}$.

In this paper, we will show that the phenomenological point of view that the reheat temperature may be as low as 1 $\mathrm{MeV}$ has rich implications for particle dark matter, neutrino mass limits, axion cosmology, and baryogenesis.

The key point of our considerations is that reheating is not an instantaneous process. On the contrary, the radiationdominated phase follows a prolonged stage of matter domination during which the energy density of the universe is dominated by the coherent oscillations of the field $\phi$. The oscillations start at time $H_{I}^{-1}$ and end when the age of the universe becomes of order of the lifetime $\Gamma_{\phi}^{-1}$ of the scalar field. At times $H_{I}^{-1} \lesssim t \lesssim \Gamma_{\phi}^{-1}$ the dynamics of the system is quite involved. During this stage the energy density per comoving volume of the $\phi$ field decreases as $\exp \left(-\Gamma_{\phi} t\right)$ and the light decay products of the scalar field thermalize. The temperature $T$ of this hot plasma, however, does not scale as $T \propto a^{-1}$ as in the ordinary radiation-dominated phase ( $a$ is the Friedmann-Robertson-Walker scale factor) [7-9], but reaches a maximum $T_{\mathrm{MAX}} \sim\left(H_{I} M_{P l}\right)^{1 / 4} T_{R H}^{1 / 2}\left(M_{P l}\right.$ is the Planck mass) and then decreases as $T \propto a^{-3 / 8}$, signaling the continuous release of entropy from the decays of the scalar field. This scaling continues until the time $t \sim \Gamma_{\phi}^{-1}$ when the radiation-dominated phase commences with temperature $T_{R H}$. Therefore, before reheating is completed, for a given temperature the universe expands faster than in the radiationdominated phase. Notice that $T_{R H}$ is not the maximum temperature during the reheat process. On the contrary, $T_{\mathrm{MAX}}$ can be much larger than $T_{R H}$. The behavior of the universe during reheating is discussed in detail in Sec. II.

In Sec. III we use these results to compute the relic abundance of a dark-matter species $(X)$ produced during reheating. We consider the case that $T_{R H}$ is smaller than the $X$ freeze-out temperature. ${ }^{2}$ Although naively one might expect a negligible $X$ number density under these circumstances, we find that the $X$ relic density can reach cosmologically interesting values. We also show that because of entropy release during the reheat stage, for a given mass and cross section the present $X$ abundance is smaller than obtained assuming freeze out in the radiation-dominated era. This relaxes the bounds coming from requiring that $\Omega_{X} h^{2} \lesssim 1$. Moreover, the parametric dependence on the mass and the annihilation cross section of the present abundance is nonstandard. Therefore, most of the cosmological constraints on specific particle

\footnotetext{
${ }^{2}$ For a different perspective, see Ref. [10].
}

properties have to be revisited.

The value of the maximum temperature during reheating, $T_{\mathrm{MAX}}$, delineates different regions for our results. If $T_{\mathrm{MAX}}$ is smaller than the $X$ mass, $X$ particles generated by collisions in the thermal bath during the coherent $\phi$ oscillations are always nonrelativistic. If the cross section is small enough, the $X$ particles do not reach chemical equilibrium and the present abundance is proportional to the cross section, $\Omega_{X}$ $\propto\left\langle\sigma_{A}|v|\right\rangle$, in contrast with the usual radiation-dominated case in which $\Omega_{X} \propto\left\langle\sigma_{A}|v|\right\rangle^{-1}$. Avoiding overclosure of the universe imposes a lower bound on the annihilation cross section. On the other hand, if the $X$ particles reach chemical equilibrium before reheating is completed, the ratio of the $X$ number density to entropy density in a comoving volume does not stop decreasing when the particles freeze out because entropy is released until reheating is over. Therefore, the present abundance does not depend only on $\left\langle\sigma_{A}|v|\right\rangle^{-1}$ (as in the standard case), but also on the reheat temperature $T_{R H}$ : the lower $T_{R H}$, the smaller the abundance. We will provide a formula for $\Omega_{X}$ which reproduces both the standard result when $T_{R H}$ is equal to the freeze-out temperature of the relic particle and the nonstandard result $\Omega_{X} \propto\left\langle\sigma_{A}|v|\right\rangle$ when the value cross section becomes smaller than some critical value.

If $T_{\mathrm{MAX}}$ and $T_{R H}$ are larger than the $X$ mass, we will show that the relevant processes determining the present $X$ abundance occur during reheating or afterwards.

An important result is that $T_{\mathrm{MAX}}$ (or equivalently $H_{I}$ ) is relevant when deciding if $X$ is relativistic or not, but does not appear in the final expression for the relic abundance. Therefore, once $T_{\mathrm{MAX}}$ has determined the pertinent case, $\Omega_{X}$ depends on the physics of the $\phi$ field only through $T_{R H}$. It is easy to understand why. The $X$ number density results from the competition of two rates, the interaction rate and the expansion rate of the universe. Before reheating is completed the expansion rate depends only on $T_{R H}, H \sim T^{4} / T_{R H}^{2} M_{P l}$, and therefore the final abundance depends only upon $T_{R H}$.

In Sec. IV we discuss the applications of our findings to some popular cold dark matter candidates. Here, we preview some of our results.

While excluded in the usual scenario, thermal weakly interactive massive particles (WIMPs) with mass larger than the unitarity bound of a few hundred $\mathrm{TeV}$ [11] may be viable dark matter candidates in low reheat models.

Of the many WIMP candidates, the best motivated seems to be the neutralino, the lightest supersymmetric particle (LSP) [12]. In the case in which the LSP is mainly a Bino, requiring $\Omega_{B} \leqslant 1$ gives an upper bound on the slepton mass $\tilde{m}_{l_{R}}[13-15]$. However, once we relax the assumption that the reheat temperature is higher than the freeze-out temperature of the WIMP, we will show that the upper bound on $\tilde{m}_{l_{R}}$ is drastically relaxed and may completely disappear. The same argument can be applied to other supersymmetric candidates.

Another striking application for dark matter that illustrates our point is the computation of the relic abundance of massive neutrinos. The well-known cosmological CowsikMcClelland-Lee-Weinberg bound $[16,17]$ rules out neutri- 
nos more massive than roughly $90 \mathrm{eV}$ and lighter than around $2 \mathrm{GeV}$. This result has a significant impact in cosmology, ruling out, for instance, the possibility that neutrinos are warm dark matter. This standard result, however, assumes that the reheat temperature is much higher than 1 $\mathrm{MeV}$ and that neutrinos have been in chemical equilibrium up to temperatures of the order of $1 \mathrm{MeV}$. We will show that if the reheat temperature is as small as allowed by big-bang nucleosynthesis, then massive, stable Dirac (Majorana) neutrinos are compatible with cosmology if they are lighter than about $33 \mathrm{keV}$ or heavier than about 6 (5) MeV. This implies, for instance, that neutrinos may still be warm or cold dark matter and play a significant role in other cosmological or astrophysical phenomena.

We then proceed by investigating the implications of a low reheat temperature for axion cosmology. It is well known that in the standard scenario the oscillations of the axion field generated by the misalignment mechanism overclose the universe unless the Peccei-Quinn scale, $f_{P Q}$, is smaller than about $10^{12} \mathrm{GeV}$. This bound, however, is obtained assuming that the reheat temperature is larger than the QCD scale. When this assumption is abandoned, the cosmological upper bound on $f_{P Q}$ is significantly relaxed to $f_{P Q}$ $\lesssim 10^{16} \mathrm{GeV}$ if $T_{R H} \sim 1 \mathrm{MeV}$.

If the $X$ particle has a nonvanishing but small decay lifetime $\tau_{X}$, its decay products may destroy the light elements generated during primordial nucleosynthesis. This gives strong constraints in the plane $\left(M_{X}, \tau_{X}\right)$ [18] that are very sensitive to the number density of the species $X$ at freeze out. Lowering the reheat temperature implies a smaller number density and therefore much less restrictive bounds.

These results all imply that presently stated cosmological limits may not always be relevant in limiting particle properties such as the supersymmetric mass spectra in the experimentally verifiable range of future colliders.

As a last application, in Sec. V we analyze the production of unstable superheavy states during the process of reheating, keeping in mind the possibility that the subsequent decay of these states may generate the observed baryon asymmetry. The fact that $T_{\mathrm{MAX}}$ is larger than the reheat temperature may give rise to an efficient production of these superheavy states. As a result, out-of-equilibrium grand unified theory (GUT) baryogenesis and/or leptogenesis can take place even if the reheat temperature is much smaller than the mass of the superheavy decaying particle. This is particularly useful in supersymmetric scenarios where $T_{R H}$ has to be low enough to avoid the overproduction of gravitinos and other dangerous relics.

Finally, Sec. VI summarizes our results.

\section{THE DYNAMICS OF REHEATING}

\section{A. The relevant Boltzmann equations}

In this section we study the Boltzmann equations for the time evolution of a system whose energy density is in the form of unstable massive particles $\phi$, stable massive particles $X$, and radiation $R$ (other similar studies can be found in Refs. $[7-9,19-21])$. We assume that $\phi$ decays into radiation with a rate $\Gamma_{\phi}$, and that the $X$ particles are created and annihilate into radiation with a thermal-averaged cross section times velocity $\langle\sigma v\rangle$. The corresponding energy and number densities satisfy the differential equations [7]

$$
\begin{aligned}
& \frac{d \rho_{\phi}}{d t}=-3 H \rho_{\phi}-\Gamma_{\phi} \rho_{\phi}, \\
& \frac{d \rho_{R}}{d t}=-4 H \rho_{R}+\Gamma_{\phi} \rho_{\phi}+\langle\sigma v\rangle 2\left\langle E_{X}\right\rangle\left[n_{X}^{2}-\left(n_{X}^{e q}\right)^{2}\right], \\
& \frac{d n_{X}}{d t}=-3 H n_{X}-\langle\sigma v\rangle\left[n_{X}^{2}-\left(n_{X}^{e q}\right)^{2}\right] .
\end{aligned}
$$

Here, we assume that each $X$ has energy $\left\langle E_{X}\right\rangle \simeq \sqrt{M^{2}+9 T^{2}}$ and the factor $2\left\langle E_{X}\right\rangle$ is the average energy released in $X$ annihilation. Later we will assume $\rho_{X}=\left\langle E_{X}\right\rangle n_{X}$. The Hubble expansion parameter $H$ is given by

$$
H^{2}=\frac{8 \pi}{3 M_{P l}^{2}}\left(\rho_{\phi}+\rho_{R}+\rho_{X}\right)
$$

The equilibrium number density for particles obeying Maxwell-Boltzmann statistics can be expressed in terms of $K_{2}$, the modified Bessel function of the second kind:

$$
\begin{aligned}
n_{X}^{e q} & =\frac{g T^{3}}{2 \pi^{2}}\left(\frac{M_{X}}{T}\right)^{2} K_{2}\left(M_{X} / T\right) \\
& \rightarrow \frac{g T^{3}}{\pi^{2}} \quad(T \gg M) \\
& \rightarrow g\left(\frac{M_{X} T}{2 \pi}\right)^{3 / 2} \exp \left(-M_{X} / T\right) \quad(T \ll M),
\end{aligned}
$$

where $g$ is the number of degrees of freedom of the $X$-particle species.

For cosmological considerations it is more appropriate to express $\Gamma_{\phi}$ in terms of the reheat temperature $T_{R H}$ using the conventional expression

$$
\Gamma_{\phi}=\sqrt{\frac{4 \pi^{3} g_{*}\left(T_{R H}\right)}{45}} \frac{T_{R H}^{2}}{M_{P l}},
$$

where $g_{*}(T)$ describes the effective number of degrees of freedom at temperature $T$. This expression defines $T_{R H}$.

Next, we express Eqs. (1)-(3) in terms of dimensionless variables and convert time derivatives to derivatives with respect to the scale factor $a$. The dimensionless variables we choose are

$$
\Phi \equiv \rho_{\phi} T_{R H}^{-1} a^{3} ; \quad R \equiv \rho_{R} a^{4} ; \quad X \equiv n_{X} a^{3} ; \quad A \equiv a / a_{I} .
$$

The choice of $T_{R H}^{-1}$ in the definition of $\Phi$ is for convenience; any mass scale would suffice. The factor $a_{I}$ will be chosen as the initial value of the scale factor for the integration. Since no physical result can depend upon the choice of $a_{I}$, we are free to choose 


$$
a_{I}=T_{R H}^{-1}
$$

In terms of the new variables, Eqs. (1)-(3) become

$$
\begin{aligned}
\frac{d \Phi}{d A}= & -\left(\frac{\pi^{2} g_{*}}{30}\right)^{1 / 2} \frac{A^{1 / 2} \Phi}{\sqrt{\Phi+R / A+X\left\langle E_{X}\right\rangle / T_{R H}}} \\
\frac{d R}{d A}= & \left(\frac{\pi^{2} g_{*}}{30}\right)^{1 / 2} \frac{A^{3 / 2} \Phi}{\sqrt{\Phi+R / A+X\left\langle E_{X}\right\rangle / T_{R H}}} \\
& +\left(\frac{3}{8 \pi}\right)^{1 / 2} \frac{A^{-3 / 2}\langle\sigma v\rangle 2\left\langle E_{X}\right\rangle M_{P l}}{\sqrt{\Phi+R / A+X\left\langle E_{X}\right\rangle / T_{R H}}}\left(X^{2}-X_{e q}^{2}\right),
\end{aligned}
$$

$$
\frac{d X}{d A}=-\left(\frac{3}{8 \pi}\right)^{1 / 2} \frac{A^{-5 / 2}\langle\sigma v\rangle M_{P l} T_{R H}}{\sqrt{\Phi+R / A+X\left\langle E_{X}\right\rangle / T_{R H}}}\left(X^{2}-X_{e q}^{2}\right) .
$$

At early times the energy density of the universe is completely dominated by the $\phi$ field. The initial value of the $\phi$ energy density can be expressed in terms of the initial expansion rate, $H_{I}$, as $\rho_{\phi}=(3 / 8 \pi) M_{P l}^{2} H_{I}^{2}$. Therefore, we will solve Eqs. (9)-(11) choosing the following initial conditions:

$$
\Phi_{I}=\frac{3}{8 \pi} \frac{M_{P l}^{2} H_{I}^{2}}{T_{R H}^{4}}, \quad R_{I}=X_{I}=0, \quad A_{I}=1 .
$$

\section{B. The temperature-scale factor relation}

During the epoch between the initial time, $H_{I}^{-1}$, and the completion of reheating at time $\Gamma_{\phi}^{-1}$, the temperature of the universe does not scale as $T \sim a^{-1}$ as in the radiationdominated era, but follows a different law [7]. This unusual relation between the temperature and the scale factor, derived below, will significantly affect the calculation of the relic abundance of $X$ particles.

The temperature of the system is measured by the radiation energy density, and therefore $T$ is related to $R$ by

$$
T=\left[\frac{30}{\pi^{2} g_{*}(T)}\right]^{1 / 4} \frac{R^{1 / 4}}{A} T_{R H} .
$$

At early times $\left(H \gg \Gamma_{\phi}\right)$, we can approximate the right-hand side of Eq. (10) by retaining only the terms proportional to the $\phi$ energy density and by taking $\Phi \simeq \Phi_{I}$. The solution of Eq. (10) then becomes

$$
R \simeq \frac{2}{5}\left(\frac{\pi^{2} g_{*}}{30}\right)^{1 / 2}\left(A^{5 / 2}-1\right) \Phi_{I}^{1 / 2}
$$

Using this result in Eq. (13) we obtain the expression for the temperature $T$ as a function of the scale factor

$$
T=T_{\text {MAX }} f(A),
$$

where $T_{\text {MAX }}$ and $f(A)$ are given by

$$
\begin{aligned}
T_{\mathrm{MAX}} \equiv & \left(\frac{3}{8}\right)^{2 / 5}\left(\frac{5}{\pi^{3}}\right)^{1 / 8} \frac{g_{*}^{1 / 8}\left(T_{R H}\right)}{g_{*}^{1 / 4}\left(T_{\mathrm{MAX}}\right)} M_{P l}^{1 / 4} H_{I}^{1 / 4} T_{R H}^{1 / 2} \\
= & {\left[\frac{g_{*}\left(T_{R H}\right)}{10}\right]^{1 / 8}\left[\frac{10}{g_{*}\left(T_{\mathrm{MAX}}\right)}\right]^{1 / 4}\left(\frac{H_{I}}{\mathrm{eV}}\right)^{1 / 4} } \\
& \times\left(\frac{T_{R H}}{100 \mathrm{MeV}}\right)^{1 / 2} 42 \mathrm{GeV} \\
f(A) \equiv & \kappa\left(A^{-3 / 2}-A^{-4}\right)^{1 / 4} .
\end{aligned}
$$

The constant $\kappa$ is defined as

$$
\kappa \equiv\left(\frac{8^{8}}{3^{3} 5^{5}}\right)^{1 / 20} \frac{g_{*}^{1 / 4}\left(T_{\mathrm{MAX}}\right)}{g_{*}^{1 / 4}(T)}=1.3 \frac{g_{*}^{1 / 4}\left(T_{\mathrm{MAX}}\right)}{g_{*}^{1 / 4}(T)} .
$$

The function $f(A)$ starts as zero, then grows until $A_{0}$ $=(8 / 3)^{2 / 5}$, where it reaches its maximum $f\left(A_{0}\right)=1$ (corresponding to $T=T_{\mathrm{MAX}}$ ), and then decreases as $A^{-3 / 8}$. Therefore, for $A>A_{0}$, Eq. (15) can be approximated by

$$
T \simeq \kappa T_{\mathrm{MAX}} A^{-3 / 8}=\left[\frac{9 g_{*}\left(T_{R H}\right)}{5 \pi^{3} g_{*}^{2}(T)}\right]^{1 / 8} M_{P l}^{1 / 4} H_{I}^{1 / 4} T_{R H}^{1 / 2} A^{-3 / 8} .
$$

This result shows that during the phase before reheating the temperature has a less steep dependence on the scale factor than in the radiation-dominated era. In other words, as the temperature decreases, the universe expands faster before reheating than in the radiation-dominated epoch. Notice also that $T_{\text {MAX }}$ can be much larger than $T_{R H}$, as long as $H_{I}$ $>T_{R H}^{2} / M_{P l}$.

\section{The temperature-expansion rate relation}

Next, consider the temperature dependence of the expansion rate $H$ during the epoch of reheating. Between the time when $T_{\text {MAX }}$ is obtained and the decay time $\Gamma_{\phi}^{-1}$, the scalar field energy density scales as $\rho_{\phi}=\Phi_{I} T_{R H}^{4} A^{-3}$. Since $H^{2}$ $\simeq(8 \pi / 3) \rho_{\phi} / M_{P l}^{2}$, we can express $H$ as

$$
H^{2}=\frac{8 \pi}{3} \frac{\Phi_{I} T_{R H^{A}}^{4}}{M_{P l}^{2}} .
$$

Now we can use Eq. (18) to express $A$ in terms of $T$, with the result

$$
H=\left[\frac{5 \pi^{3} g_{*}^{2}(T)}{9 g_{*}\left(T_{R H}\right)}\right]^{1 / 2} \frac{T^{4}}{T_{R H}^{2} M_{P l}} .
$$

This result can be compared to the result for a radiationdominated universe $\left(H \propto T^{2}\right)$ and a matter-dominated universe $\left(H \propto T^{3 / 2}\right)$.

\section{CALCULATION OF THE RELIC ABUNDANCE}

In this paper we are interested in the situation in which the $X$ particles never obtain chemical equilibrium in the 
radiation-dominated era after reheating. ${ }^{3}$ This means that $T_{R H}$ must be smaller than the conventional freeze-out temperature (roughly equal to $M_{X} / 20$ for nonrelativistic, weakly interacting particles). In this situation we can encounter several possibilities. In the first case, the $X$ particles are always nonrelativistic and never in chemical equilibrium, either before or after reheating. In the second case, the nonrelativistic $X$ particles reach chemical equilibrium, but then freeze out before the completion of the reheat process. Finally, we can consider the case when the relevant processes of particle production and freeze out occur before reheating at temperatures at which $X$ is still relativistic. In this section, we will compute the $X$ thermal relic abundance in all these cases.

In principle, the relic abundance could receive contributions from other sources, like the direct $\phi$ decay into $X$ particles [22], or from the production and decay of heavy particles eventually decaying into $X$. In this paper we will ignore these model-dependent effects and therefore our calculation can be viewed as a lower bound on the $X$ abundance.

The nonrelativistic and relativistic cases are discriminated by the conditions $M_{X}>T_{\mathrm{MAX}}$ and $M_{X}<T_{\mathrm{MAX}}$, respectively. This translates into a condition on $H_{I}$; for instance, the nonrelativistic case corresponds to

$$
\begin{aligned}
H_{I}< & \left(\frac{8}{3}\right)^{8 / 5}\left(\frac{\pi^{3}}{5}\right)^{1 / 2} \frac{g_{*}\left(T_{\mathrm{MAX}}\right)}{g_{*}^{1 / 2}\left(T_{R H}\right)} \frac{M_{X}^{4}}{M_{P l} T_{R H}^{2}} \\
= & {\left[\frac{g_{*}\left(T_{\mathrm{MAX}}\right)}{10}\right]\left[\frac{10}{g_{*}\left(T_{R H}\right)}\right]^{1 / 2}\left(\frac{M_{X}}{100 \mathrm{GeV}}\right)^{4} } \\
& \times\left(\frac{100 \mathrm{MeV}}{T_{R H}}\right)^{2} 31 \mathrm{eV} .
\end{aligned}
$$

In this paper we will treat $T_{R H}$ and $T_{\mathrm{MAX}}$ as free parameters and we will not rely on particular models of inflation or $\phi$ decay. Nevertheless, it is useful to show what kind of $\phi$ physics can give rise to the different cases considered in this section. First of all, we are interested in very low reheat temperatures. This can be achieved if $\phi$ has a typical gravitational decay width $\Gamma_{\phi} \sim M_{\phi}^{3} / M_{P l}^{2}$, for which

$$
T_{R H} \sim\left(\frac{10}{g_{*}}\right)^{1 / 4}\left(\frac{M_{\phi}}{100 \mathrm{TeV}}\right)^{3 / 2} 4 \mathrm{MeV} .
$$

The value of $T_{\mathrm{MAX}}$ is determined by the initial $\phi$ energy density. If $M_{\phi}$ is the mass scale characterizing the physics of $\phi$, we can expect $\rho_{\phi}\left(a_{I}\right) \sim M_{\phi}^{4}$. This happens, for instance, in hybrid models of inflation. In this case we find

\footnotetext{
${ }^{3}$ Here we make the usual distinction between chemical equilibrium and kinetic equilibrium. Kinetic equilibrium can be achieved by $X$-number conserving scatterings, such as $\gamma X \leftrightarrow \gamma X$ ( $\gamma$ represents a light degree of freedom like a photon). Chemical equilibrium can only be achieved by processes that change the number of $X$ particles, such as $X X \leftrightarrow \gamma \gamma$. For massive particles the cross section for the second process may be orders of magnitude smaller than the cross section for the first process, and it is possible to assume kinetic equilibrium but not chemical equilibrium.
}

$$
T_{\mathrm{MAX}} \sim\left(\frac{10}{g_{*}}\right)^{1 / 4}\left(\frac{M_{\phi}}{100 \mathrm{TeV}}\right)^{5 / 4} 10 \mathrm{GeV}
$$

On the other hand, in the case of chaotic inflation, one expects that the $\phi$ field has an initial value of the order of the Planck mass and therefore $\rho_{\phi}\left(a_{I}\right) \sim M_{\phi}^{2} M_{P l}^{2}$. This leads to

$$
T_{\mathrm{MAX}} \sim\left(\frac{10}{g_{*}}\right)^{1 / 4}\left(\frac{M_{\phi}}{100 \mathrm{TeV}}\right) 30 \mathrm{TeV}
$$

For an $X$ particle with typical electroweak mass and for $M_{\phi} \sim 100 \mathrm{TeV}$, the two options correspond to the nonrelativistic and relativistic case, respectively.

\section{A. Nonrelativistic nonequilibrium production and freeze out}

Let us suppose that the $X$ particles are always nonrelativistic and the condition in Eq. (21) is satisfied. Since we are considering the case in which $X$ does not reach chemical equilibrium $\left(X \ll X_{e q}\right)$, at early times, Eq. (11) can be approximated by

$$
\frac{d X}{d A}=\left(\frac{3}{8 \pi}\right)^{1 / 2} A^{-5 / 2}\langle\sigma v\rangle M_{P l} T_{R H} X_{e q}^{2} \Phi_{I}^{-1 / 2} .
$$

The equilibrium distribution in the nonrelativistic limit is

$$
X_{e q}=g A^{3}\left(\frac{M_{X} T}{2 \pi T_{R H}^{2}}\right)^{3 / 2} \exp \left(-M_{X} / T\right),
$$

where $g$ is the number of degrees of freedom of the particle $X$ and the temperature $T$ is given by Eq. (18). We express the thermal-averaged annihilation cross section times velocity as

$$
\langle\sigma v\rangle \equiv \frac{1}{M_{X}^{2}}\left(\alpha_{s}+\frac{T}{M_{X}} \alpha_{p}\right) .
$$

Here the dimensionless coefficients $\alpha_{s}$ and $\alpha_{p}$ describe, respectively, the $s$-wave and $p$-wave annihilations in a nonrelativistic expansion of the cross section. Using Eqs. (12), (18), (26), and (27) in Eq. (25), we obtain

$$
\begin{aligned}
\frac{d X}{d A}= & \frac{g^{2}}{(2 \pi)^{3}} \frac{M_{X} \kappa^{3} T_{\mathrm{MAX}}^{3}}{H_{I} T_{R H}^{3}} \exp \left(-\frac{2 M_{X}}{\kappa T_{\mathrm{MAX}}} A^{3 / 8}\right) \\
& \times\left(\alpha_{s} A^{19 / 8}+\alpha_{p} A^{2} \frac{\kappa T_{\mathrm{MAX}}}{M_{X}}\right) .
\end{aligned}
$$

We will find the solution for $X(\infty)$ as a Gaussian-integral approximation to Eq. (28). Although Eq. (28) is valid only at early times, we will integrate it in the full range between $A$ $=0$ and $A=\infty$. This is a good approximation because the exponential suppression makes the right-hand side of Eq. (28) negligible anywhere outside a small interval of scale factors centered around $A=A_{*}$, with

$$
A_{*}=\left(\frac{17}{2} \frac{\kappa T_{\mathrm{MAX}}}{2 M_{X}}\right)^{8 / 3} \text { for } s \text { wave, }
$$




$$
A_{*}=\left(\frac{15}{2} \frac{\kappa T_{\mathrm{MAX}}}{2 M_{X}}\right)^{8 / 3} \text { for } p \text { wave. }
$$

Using Eq. (18), we find that $A_{*}$ corresponds to a temperature $T_{*}=4 M_{X} / 17$ for the $s$-wave and $T_{*}=4 M_{X} / 15$ for the $p$-wave. Therefore, $T_{*}$ is the temperature at which most of the $X$-particle production takes place. We will assume $T_{*}$ $<T_{\text {MAX }}$ or else the final $X$-particle density is suppressed by a very small exponential function. The Gaussian-integral approximation to Eq. (28) is ${ }^{4}$

$$
\begin{aligned}
X_{\infty}= & \frac{8 g^{2} M_{X}^{4} \sqrt{2 \pi}}{3 \pi^{3} H_{I} T_{R H}^{3}}\left(\frac{\kappa T_{\mathrm{MAX}}}{2 M_{X}}\right)^{12} \\
& \times \exp (-17 / 2)\left(\frac{17}{2}\right)^{17 / 2}\left(\alpha_{s}+\frac{\alpha_{p}}{4}\right) .
\end{aligned}
$$

Next we want to relate $X_{\infty}$ to the mass density of $X$ particles today. After particle production stops at $A \simeq A_{*}$, the factor $X \propto n_{X} A^{3}=X_{\infty}$ remains constant. Therefore, at reheating

$$
\rho_{X}\left(T_{R H}\right)=M_{X} n_{X}\left(T_{R H}\right)=M_{X} X_{\infty} T_{R H}^{3} A_{R H}^{-3},
$$

where from Eq. (18)

$$
A_{R H}^{-3}=\left(\frac{T_{R H}}{\kappa T_{\mathrm{MAX}}}\right)^{8}=\frac{5 \pi^{3} g_{*}\left(T_{R H}\right) T_{R H}^{4}}{9 M_{P l}^{2} H_{I}^{2}} .
$$

Also, at reheating the radiation energy density is

$$
\rho_{R}\left(T_{R H}\right)=\frac{\pi^{2}}{30} g_{*}\left(T_{R H}\right) T_{R H}^{4} .
$$

After the completion of reheating the universe is radiation dominated, and

$$
\frac{\rho_{X}\left(T_{\text {now }}\right)}{\rho_{R}\left(T_{\text {now }}\right)}=\frac{T}{T_{\text {now }}} \frac{\rho_{X}(T)}{\rho_{R}(T)} .
$$

Of course the extraction of energy from the scalar field is not an instantaneous process, but we can use $T=T_{R H}$ in Eq. (35) and correct for the entropy release after $T_{R H}$. It is straightforward to demonstrate (see the Appendix) that relatively independent of the model parameters, only about $25 \%$ of the comoving $\phi$ energy density has been extracted at $T=T_{R H}$. At temperatures smaller than $T_{R H}$ some residual entropy is released by the decays of the scalar field till the time when the energy density in radiation significantly dominates over the energy density of the scalar field. One can show that (again nearly independent of model parameters) the comov-

\footnotetext{
${ }^{4}$ The result of the Gaussian integration in Eq. (31) is a very good approximation of the exact integral, which is given by $\quad X_{\infty}=\left(8 g^{2} M_{X}^{5}\right) /\left(3 \pi^{3} H_{I}\right)\left[\alpha_{s} f_{s}\left(2 M_{X} / \kappa T_{\max }\right)+\alpha_{p} f_{p}\left(2 M_{X} /\right.\right.$ $\left.\left.\kappa T_{\max }\right)\right]$, where $\quad f_{s}(x)=\exp (-x) 8 ! \Sigma_{k=0}^{8} x^{k-12} / k ! \simeq \sqrt{2 \pi}(17 /$ 2) ${ }^{17 / 2} \exp (-17 / 2) x^{-12}$ and $f_{p}(x)=\exp (-x)(8 ! / 4) \sum_{k=0}^{7} x^{k-12} / k$ ! $\simeq 2 \sqrt{2 \pi}(15 / 2)^{15 / 2} \exp (-15 / 2) x^{-12} \simeq f_{s}(x) / 4$.
}

ing entropy increases by about a factor of 8 after $T_{R H}$. Therefore all the analytic estimates should be divided by a factor of 8 . This is confirmed by numerical calculations as shown in Sec. III E. The reader is referred to the Appendix for more details.

From Eq. (35) with $T=T_{R H}$ and the extra factor of $1 / 8$, we obtain an estimate for the present energy density of $X$ particles in units of the critical density

$$
\begin{aligned}
\Omega_{X} h^{2}= & \frac{3 \sqrt{10}(17 / 2 e)^{17 / 2}}{2048 \pi^{6}} \frac{g^{2} g_{*}^{3 / 2}\left(T_{R H}\right)}{g_{*}^{3}\left(T_{*}\right)} \\
& \times \frac{M_{P l} T_{R H}^{7}}{M_{X}^{7} T_{\text {now }}}\left(\alpha_{s}+\alpha_{p} / 4\right) \Omega_{R} h^{2} \\
= & 2.1 \times 10^{4}\left(\frac{g}{2}\right)^{2}\left[\frac{g_{*}\left(T_{R H}\right)}{10}\right]^{3 / 2}\left[\frac{10}{g_{*}\left(T_{*}\right)}\right]^{3} \\
& \times\left(\frac{10^{3} T_{R H}}{M_{X}}\right)^{7}\left(\alpha_{s}+\alpha_{p} / 4\right)
\end{aligned}
$$

(nonrelativistic nonequilibrium

production during reheating era).

Here we have used $T_{\text {now }}=2.35 \times 10^{-13} \mathrm{GeV}$ and $\Omega_{R} h^{2}$ $=4.17 \times 10^{-5}$, including the contributions from the cosmic background radiation and from neutrinos.

Notice that in this case $\Omega_{X}$ is proportional to the annihilation cross section, instead of being inversely proportional, as in the case of the usual thermal relic abundance calculation in a radiation-dominated universe.

The basic assumption used in this section is that the $X$ particles never reach thermal equilibrium. This hypothesis holds if at the time of maximum particle production the $X$ number density is less than the equilibrium value, $X_{\infty}$ $<X_{e q}\left(T_{*}\right)$. Using Eqs. (26) and (31), we find that this condition corresponds to a limit on the annihilation cross section $\alpha_{s}<\bar{\alpha}_{s}$ for the $s$-wave or $\alpha_{p}<\bar{\alpha}_{p}$ for the $p$-wave, with

$$
\begin{aligned}
\bar{\alpha}_{s}= & \frac{4 \sqrt{10} \pi^{5 / 2} e^{17 / 4}}{289} \frac{g_{*}\left(T_{*}\right)}{g g_{*}^{1 / 2}\left(T_{R H}\right)} \frac{M_{X}^{3}}{M_{P l} T_{R H}^{2}} \\
= & 7 \times 10^{-10} \frac{2}{g}\left[\frac{g_{*}\left(T_{*}\right)}{10}\right]\left[\frac{10}{g_{*}\left(T_{R H}\right)}\right]^{1 / 2}\left(\frac{M_{X}}{100 \mathrm{GeV}}\right)^{3} \\
& \times\left(\frac{100 \mathrm{MeV}}{T_{R H}}\right)^{2}, \\
\bar{\alpha}_{p}= & \frac{2 \sqrt{10} \pi^{5 / 2} e^{15 / 4}}{15} \frac{g_{*}\left(T_{*}\right)}{g g_{*}^{1 / 2}\left(T_{R H}\right)} \frac{M_{X}^{3}}{M_{P l} T_{R H}^{2}} \\
= & 4 \times 10^{-9} \frac{2}{g}\left[\frac{g_{*}\left(T_{*}\right)}{10}\right]\left[\frac{10}{g_{*}\left(T_{R H}\right)}\right]^{1 / 2}\left(\frac{M_{X}}{100 \mathrm{GeV}}\right)^{3} \\
& \times\left(\frac{100 \mathrm{MeV}}{T_{R H}}\right)^{2} \cdot
\end{aligned}
$$


In the nonequilibrium case considered in this section, we find that there is a maximum value of $\Omega_{X}$ that can be achieved. This is obtained by replacing the constraint $\alpha_{s}$ $<\bar{\alpha}_{s}$ into Eq. (36),

$$
\begin{aligned}
\Omega_{X} h^{2}< & 1 \times 10^{-5} \frac{g}{2}\left[\frac{g_{*}\left(T_{R H}\right)}{10}\right]\left[\frac{10}{g_{*}\left(T_{*}\right)}\right]^{2} \\
& \times\left(\frac{T_{R H}}{100 \mathrm{MeV}}\right)^{5}\left(\frac{100 \mathrm{GeV}}{M_{X}}\right)^{4} .
\end{aligned}
$$

A similar constraint can be obtained in the case of $p$-wave annihilation.

To conclude this section, we want to show that for the case under consideration the process of particle production always freezes out before reheating, i.e.,

$$
H\left(A_{*}\right)>\Gamma_{\phi} .
$$

Since the universe is matter dominated by the $\phi$ field, $H$ scales like $A^{-3 / 2}$ and therefore $H\left(A_{*}\right)=H_{I} A_{*}^{-3 / 2}$. Using Eqs. (29),(30), we find that the relation in Eq. (40) is satisfied whenever $M_{X}>3 T_{R H}$. This condition is always verified once we assume that the $X$ particles do not thermalize after reheating.

\section{B. Nonrelativistic equilibrium production and freeze out}

We will now consider the case in which the annihilation cross section is large $\left(\alpha_{s}>\bar{\alpha}_{s}\right.$ or $\left.\alpha_{p}>\bar{\alpha}_{p}\right)$ and the $X$ particle species reaches chemical equilibrium before reheating (this case is also discussed in Ref. [19]). The calculation of $\Omega_{X}$ is now analogous to the ordinary calculation of thermal relic abundances. However, the result is different because the relation between temperature and scale factor is not the same as in the ordinary case of a radiation-dominated universe.

The freeze-out temperature $T_{F}$ is obtained by solving for the condition

$$
n_{X}^{e q}\left(T_{F}\right)\langle\sigma v\rangle=H\left(T_{F}\right) .
$$

Here $n_{X}^{e q}=X_{e q} A^{-3} T_{R H}^{3}$ is the equilibrium number density of $X$ particles and the expansion rate $H$ as a function of temperature was expressed in Eq. (20).

Defining $x_{F} \equiv M_{X} / T_{F}$, the condition in Eq. (41) can be written as

$$
x_{F}=\ln \left[\frac{3}{2 \sqrt{10} \pi^{3}} \frac{g g_{*}^{1 / 2}\left(T_{R H}\right)}{g_{*}\left(T_{F}\right)} \frac{M_{P l} T_{R H}^{2}}{M_{X}^{3}}\left(\alpha_{s} x_{F}^{5 / 2}+5 \alpha_{p} x_{F}^{3 / 2} / 4\right)\right] .
$$

The factor $5 / 4$ in front of the $p$-wave term has been added to match the analytic solution of the Boltzmann equation. Notice that Eq. (42) admits a solution only for $\alpha_{s}>1.5 \bar{\alpha}_{s}$ or $\alpha_{p}>0.4 \bar{\alpha}_{p}$, in nice agreement with the starting assumption on the annihilation cross section.

For comparison, we remind the reader that in the case of a radiation-dominated universe the expansion rate is $H$ $=\sqrt{4 \pi^{3} g_{*} / 45} T^{2} / M_{P l}$ and the analog to Eq. (41) is

$$
x_{F}=\ln \left[\frac{3 \sqrt{5}}{4 \sqrt{2} \pi^{3}} \frac{g}{g_{*}^{1 / 2}\left(T_{F}\right)} \frac{M_{P l}}{M_{X}}\left(\alpha_{s} x_{F}^{1 / 2}+2 \alpha_{p} x_{F}^{-1 / 2}\right)\right] .
$$

In order to compute $\Omega_{X}$ we can use Eq. (35) (again with the understanding that there will be an overall correction due to the fact that the reheating process is not complete at $T_{R H}$ ) to derive the result

$$
\begin{aligned}
\rho_{X}\left(T_{R H}\right) & =\left(\frac{g_{*}\left(T_{R H}\right)}{g_{*}\left(T_{F}\right)}\right)^{2}\left(\frac{T_{R H}}{T_{F}}\right)^{8} \rho_{X}^{e q}\left(T_{F}\right) \\
& =\frac{g}{(2 \pi)^{3 / 2}}\left(\frac{g_{*}\left(T_{R H}\right)}{g_{*}\left(T_{F}\right)}\right)^{2} \frac{T_{R H}^{8}}{M_{X}^{4}} x_{F}^{13 / 2} \exp \left(-x_{F}\right) .
\end{aligned}
$$

Thus we obtain, in the case where decoupling occurs during reheating, the result

$$
\begin{aligned}
\Omega_{X} h^{2}= & \frac{5 \sqrt{5}}{4 \sqrt{\pi}} \frac{g_{*}^{1 / 2}\left(T_{R H}\right)}{g_{*}\left(T_{F}\right)} \frac{T_{R H}^{3}}{T_{\text {now }} M_{X} M_{P l}} \\
& \times \frac{1}{\left(\alpha_{s} x_{F}^{-4}+4 \alpha_{p} x_{F}^{-5} / 5\right)} \Omega_{R} h^{2} \\
= & 2.3 \times 10^{-11} \frac{g_{*}^{1 / 2}\left(T_{R H}\right)}{g_{*}\left(T_{F}\right)} \frac{T_{R H}^{3} \mathrm{GeV}^{-2}}{M_{X}\left(\alpha_{s} x_{F}^{-4}+4 \alpha_{p} x_{F}^{-5} / 5\right)}
\end{aligned}
$$

(nonrelativistic equilibrium production during reheat era).

In this case $\Omega_{X}$ is inversely proportional to the annihilation cross section, as in the radiation-dominated case. Equation (45) generalizes the ordinary result of decoupling during the radiation-dominated era, which is given by

$$
\begin{aligned}
\Omega_{X} h^{2} & =\frac{4 \sqrt{5} \Omega_{R} h^{2} M_{X}^{2}}{\sqrt{\pi} T_{\text {now }} g_{*}^{1 / 2}\left(T_{F}\right) M_{P l}\left(\alpha_{s} x_{F}^{-1}+\alpha_{p} x_{F}^{-2} / 2\right)} \\
& =7.3 \times 10^{-11} \frac{1}{g_{*}^{1 / 2}\left(T_{F}\right)} \frac{\mathrm{GeV}^{-2}}{M_{X}^{-2}\left(\alpha_{s} x_{F}^{-1}+\alpha_{p} x_{F}^{-2} / 2\right)}
\end{aligned}
$$

(nonrelativistic production during radiation era). (46)

Indeed, Eq. (45) approximately reduces to Eq. (46) as $T_{R H}$ approaches $T_{F}$. Furthermore, Eq. (45) also reproduces Eq. (36) when $\alpha_{s}$ approaches $\bar{\alpha}_{s}$ from above.

The effect of a low reheat temperature is to reduce the relic abundance with respect to the ordinary case by a factor $T_{R H}^{3} T_{F}^{\text {old }} /\left(T_{F}^{\text {new }}\right)^{4}$, where $T_{F}^{\text {old }}$ and $T_{F}^{\text {new }}$ are the freeze-out temperatures in cosmologies with high and low $T_{R H}$, respectively. This suppression factor can be understood in the following way. During the epoch before reheating, the expansion is faster than in the radiation-dominated era; freeze out occurs earlier, enhancing the $X$ abundance at $T$ $=T_{F}$ [see Eqs. (42) and (43)]. However, as the universe 

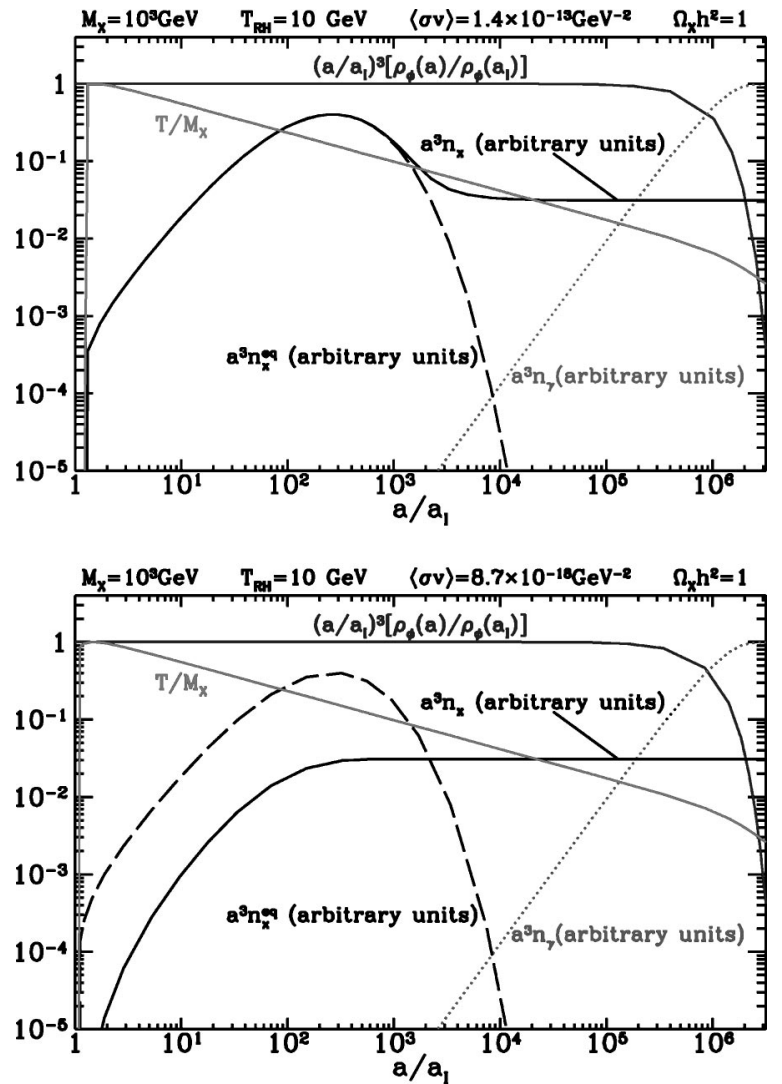

FIG. 1. Shown in the upper graph is the evolution of the $X$ density in the case where the cross section is sufficiently large to establish chemical equilibrium prior to freeze out. The lower graph illustrates the case where the cross section is too small to establish chemical equilibrium. The two cross sections were chosen to result in the same final $X$ abundances necessary to give a critical density of $X$ particles today. In the calculations $g_{*}$ was kept constant at $g_{*}=30$.

cools from $T_{F}$ to $T_{R H}$, the expansion dilutes $n_{X}$ by a factor $\left(T_{R H} / T_{F}\right)^{8}$; the dilution is more effective than in the matterdominated case $\left(n_{X} \sim T^{3}\right)$ because of entropy release during reheating. This explains why $\Omega_{X}$ is roughly $T_{R H}^{3} T_{F}^{\text {old }} /\left(T_{F}^{\text {new }}\right)^{4}$ times the relic density obtained in the case of large reheat temperature.

An illustration of the freeze out of the $X$ abundance in equilibrium and out of equilibrium is illustrated in Fig. 1. In both cases the final $X$ abundance is the same. In the top graph the cross section is large enough to establish equilibrium prior to freeze out, while in the lower graph the cross section is too small to establish equilibrium.

\section{Nonrelativistic production and freeze out}

The relic density calculations performed in Secs. III A and III B, under the assumptions of out-of-equilibrium and equilibrium, respectively, match well in the intermediate region in spite of the fact that they are derived with different approximations. Indeed, if we use in Eq. (45) the minimum allowed cross section $\left(\alpha_{s}=\bar{\alpha}_{s}\right)$, corresponding to the maximum allowed freeze-out temperature $\left(x_{F}=5 / 2\right)$, we obtain the bound

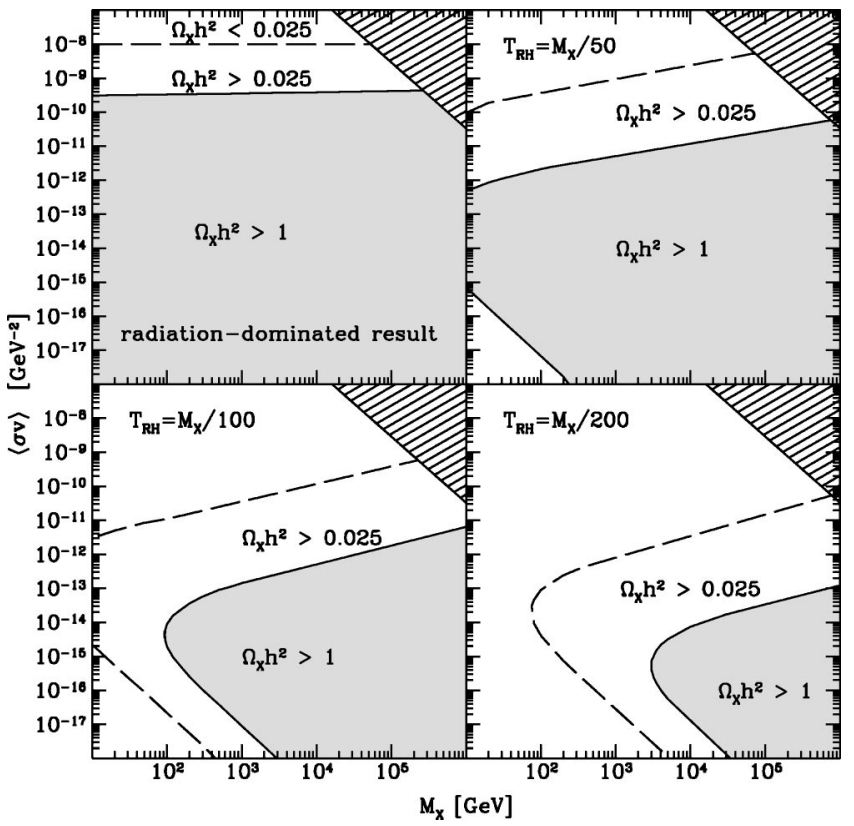

FIG. 2. The shaded areas show the cosmologically excluded regions for a particle of mass $M_{X}$ with 2 degrees of freedom which annihilates in the $s$-wave with a thermal-averaged nonrelativistic cross section $\langle\sigma v\rangle$. The upper-left figure is the usual case where particle freeze out occurs when the universe is radiation dominated. In the other frames, we have chosen $M_{X} / T_{R H}=50,100$, and 200. The interesting region for cold dark matter $\left(0.025<\Omega_{X} h^{2}<1\right)$ is between the dashed line and the shaded area. The upper right-hand corner of the $M_{X}-\langle\sigma v\rangle$ plane is excluded by unitarity arguments.

$$
\Omega_{X} h^{2}<4 \times 10^{-6} \frac{g}{2}\left[\frac{10}{g_{*}\left(T_{R H}\right)}\right]\left(\frac{T_{R H}}{100 \mathrm{MeV}}\right)^{5}\left(\frac{100 \mathrm{GeV}}{M_{X}}\right)^{4} .
$$

When this bound is saturated, we are approaching the transition from the results of Sec. III B to those of Sec. III A. Indeed, the results in Eqs. (39) and (47) turn out to be in fair agreement with each other. Similar conclusions can be obtained in the case of $p$-wave annihilation, but for simplicity in this section we will consider only the case of dominant $s$-wave annihilation.

In the ordinary case of large reheat temperature $\left(T_{R H}\right.$ $\gtrsim M_{X}$, i.e., production and freeze out in a radiationdominated universe), $\Omega_{X} h^{2}$ is proportional to $\langle\sigma v\rangle^{-1}$, as can be seen from Eq. (46). So except for a logarithmic correction, the is no explicit mass dependence to $\Omega_{X} h^{2}$. The constraint from the age of the universe, $\Omega_{X} h^{2} \lesssim 1$, implies a lower bound on the $X$ annihilation cross section, as shown in the upper-left graph in Fig. 2. The unitarity limit to $\langle\sigma v\rangle$ as a function of the mass, $\langle\sigma v\rangle_{\mathrm{MAX}}=8 \pi / M_{X}^{2}$, is also shown. The two bounds cross at the value $M_{X}=340 \mathrm{TeV}$ [11], which is usually taken as a cosmological upper bound on any stable massive particle.

In Fig. 2 we show how the cosmological bounds on $M_{X}$ can be relaxed for sufficiently low $T_{R H}$. As $T_{R H}$ is decreased, the allowed region in the parameter space $\langle\sigma v\rangle$ versus $M_{X}$ grows. The numerical results presented in the figure 


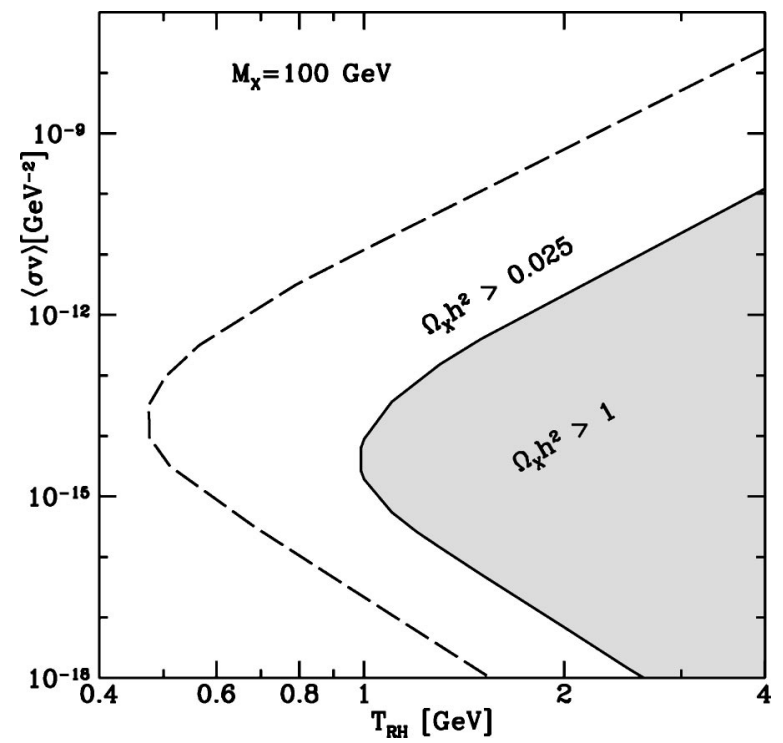

FIG. 3. The shaded region shows the cosmologically excluded region, as a function of the reheat temperature $T_{R H}$, for a particle of mass $M_{X}=100 \mathrm{GeV}$ with 2 degrees of freedom which annihilates with a thermal-averaged nonrelativistic $s$-wave cross section $\langle\sigma v\rangle$. The region, interesting for cold dark matter $\left(0.025<\Omega_{X} h^{2}<1\right)$, is delimited between the dashed line and the shaded area.

(assuming $g_{*}=30$ ) are in excellent agreement with the analytic estimates. The results are simple to understand.

In the case of low reheat temperature, the constraint $\Omega_{X} h^{2}<1$ does not simply give a lower bound on $\langle\sigma v\rangle$, but rules out a range of values. Indeed, for very small $\langle\sigma v\rangle$ we are in the limit of nonrelativistic nonequilibrium production discussed in Sec. III A, and Eq. (36) applies. For fixed $T_{R H} / M_{X}, \Omega_{X} h^{2} \propto M_{X}^{2}\langle\sigma v\rangle$, and the value of $\langle\sigma v\rangle$ forming contours of constant $\Omega_{X} h^{2}$ will scale as $M_{X}^{-2}$. As $\langle\sigma v\rangle$ grows for fixed $M_{X}, \Omega_{X}$ increases and eventually may conflict with the age of the universe constraint. After the maximum value of $\Omega_{X}$ is reached, see Eq. (39), a further increase of $\langle\sigma v\rangle$ brings us to the limit of nonrelativistic equilibrium production of Sec. III B. Now $\Omega_{X}$ is obtained from Eq. (45), and the value of $\langle\sigma v\rangle$ to give contours of fixed $\Omega_{X} h^{2}$ increases (albeit slowly) as $M_{X}$ grows.

Figure 2 also shows the parameter region in which the particle $X$ could be an interesting cold dark matter candidate, $0.025<\Omega_{X} h^{2}<1$. Values of annihilation cross sections and masses which are ordinarily excluded in the case of large $T_{R H}$ can now be of particular cosmological and observational interest.

In Fig. 3 we show the cosmologically excluded region $\left(\Omega_{X} h^{2}>1\right)$ and the region relevant for dark matter $(0.025$ $\left.<\Omega_{X} h^{2}<1\right)$ as a function of $T_{R H}$ for a fixed value $M_{X}$ $=100 \mathrm{GeV}$. Notice how the lower limit on $\langle\sigma v\rangle$, which is $2 \times 10^{-10} \mathrm{GeV}^{-2}$ in the ordinary radiation-dominated cosmology, is relaxed as $T_{R H}$ is lowered.

A striking implication of dark matter production during reheating is that the unitarity bound, $M_{X}<340 \mathrm{TeV}$, disappears and one could conceive thermal relics of very heavy particles without conflicting with the age of the universe. The necessary assumption is that $T_{\mathrm{MAX}}$ is larger than the

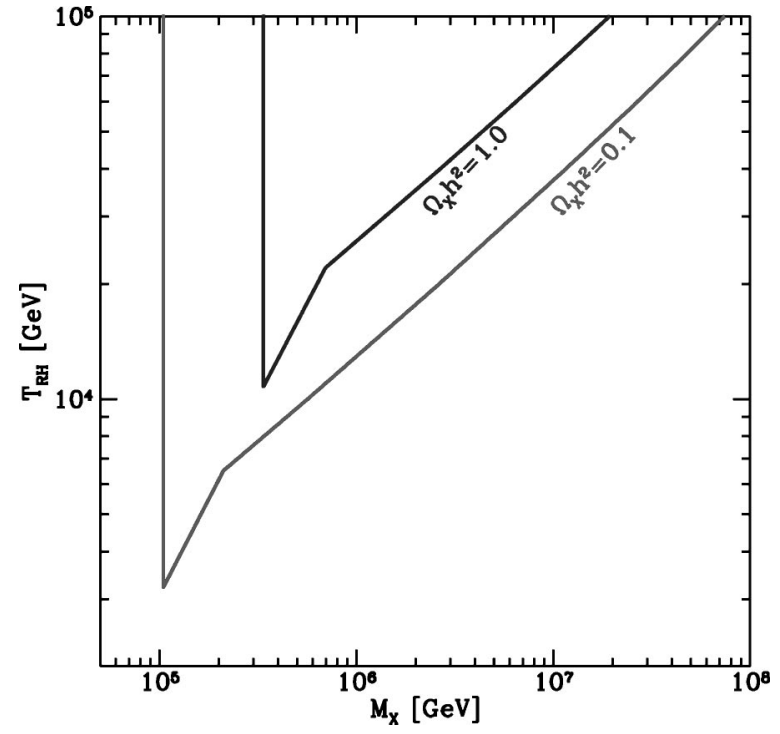

FIG. 4. The relic abundance $\Omega_{X} h^{2}$ as a function of the reheat temperature $T_{R H}$ for a particle with 2 degrees of freedom, mass $M_{X}$, and a nonrelativistic annihilation cross section in the $s$-wave saturating the unitarity bound.

temperature at which the relevant physical processes occur, i.e., $T_{*}$ in the out-of-equilibrium case and $T_{F}$ in the equilibrium case. Once this assumption is made, the final result on $\Omega_{X}$ does not depend on $T_{\mathrm{MAX}}$ or other any initial conditions of the inflationary model, but only on $T_{R H}$. Figure 4 shows how the unitarity bound is modified in the presence of low $T_{R H}$. Here we have taken $\alpha_{s}=8 \pi$ and plotted contours of various values of $\Omega_{X} h^{2}$ as a function of $M_{X}$ and $T_{R H}$.

\section{Relativistic $X$}

In this section we consider the case in which the relevant physical processes of particle production and freeze out occur when $X$ is still relativistic, and therefore we assume that the inequality of Eq. (21) is not satisfied. If the annihilation cross section is not suppressed by any mass scale larger than $M_{X}$, then $\langle\sigma v\rangle \sim T^{-2}$ and $X$ remains in thermal equilibrium until it becomes nonrelativistic. More interesting is the case in which the annihilation process depends on a new mass scale $M_{G} \gg M_{X}$. Therefore, we define

$$
\langle\sigma v\rangle \equiv \frac{T^{n}}{M_{G}^{n+2}},
$$

for a generic exponent $n$. As concrete examples, one can think of a heavy neutrino or a neutralino in the regime in which the temperature is larger than their masses: in this case $n=2$ and $M_{G}$ is roughly the mass of an intermediate gauge boson or of a slepton, respectively. Another interesting example is the gravitino, for which $n=0$ and $M_{G} \sim M_{P l}$. Finally, one can consider the graviton Kaluza-Klein excitations in theories with $\delta$ large extra dimensions [2], for which $n$ $=\delta$ and $M_{G}$ is of the order of the fundamental gravitational scale. 
Let us first consider the case in which $X$ does not reach an equilibrium density. The analysis is similar to the one performed in Sec. III A. At early times, the Boltzmann equation can be approximated by

$$
\begin{aligned}
& \frac{d X}{d A}=\sqrt{\frac{3}{8 \pi}} A^{-5 / 2}\langle\sigma v\rangle M_{P l} T_{R H} \Phi_{I}^{-1 / 2} X_{e q}^{2}, \\
& X_{e q}=\frac{c_{\xi}}{\pi^{2}}\left(\frac{T}{T_{R H}}\right)^{3} A^{3},
\end{aligned}
$$

where $c_{\xi}=g \xi(3)$ for bosons, $c_{\xi}=(3 / 4) g \xi(3)$ for fermions, and $g$ is the number of $X$ degrees of freedom. Using the relation between scale factor and temperature in Eq. (18), we can rewrite Eq. (49) as

$$
\frac{d X}{d T}=-\frac{8}{\sqrt{5 \pi^{11}}} \frac{g_{*}^{1 / 2}\left(T_{R H}\right)}{g_{*}(T)} c_{\tilde{\xi}}^{2} \frac{\left(\kappa T_{\max }\right)^{8} M_{P l}}{T^{7-n} M_{G}^{2+n} T_{R H}} .
$$

For $n<6$ the $X$-particle production dominantly occurs at the lowest possible temperature. In all interesting situations we know of, the annihilation cross section is such that $n<6$, and therefore we consider only this case. Integrating Eq. (50) up to a final temperature $T_{f}$, we obtain for $n<6$,

$$
n_{X}\left(T_{f}\right)=\frac{1}{6-n} \frac{8}{\sqrt{5 \pi^{11}}} \frac{g_{*}^{1 / 2}\left(T_{R H}\right)}{g_{*}\left(T_{f}\right)} c_{\xi}^{2}\left(\frac{T_{f}}{M_{G}}\right)^{n+2} M_{P l} T_{R H}^{2} .
$$

If $M_{X}>T_{R H}$, Eq. (51) should be evaluated at $T_{f}=M_{X}$ and the result be used as an initial condition for the nonrelativistic analysis. Since we have linearized the differential equation, within our approximation this simply amounts to adding the relic density obtained from Eq. (51) to the contribution derived in Sec. III A.

If $M_{X}<T_{R H}$, as it is usually the case for the gravitino, then in Eq. (51) we can take $T_{f}=T_{R H}$. At temperatures smaller than $T_{R H}$, the universe is radiation dominated and a calculation analogous to the one that led us to Eq. (51) shows that in this case $X$-particle production dominantly occurs at the largest possible temperature, as long as $n>-1$. Therefore all the relevant dynamics occurs at $T=T_{R H}$. The relic abundance can be obtained by rescaling the $X$ number density in Eq. (51) to the present temperature, as done in Sec. III A, to yield

$$
\begin{aligned}
\Omega_{X} h^{2} & =\frac{48 \sqrt{5}}{(6-n) \pi^{15 / 2}} \frac{c_{\xi}^{2}}{g_{*}^{3 / 2}\left(T_{R H}\right)} \frac{M_{P l} M_{X} T_{R H}^{n+1}}{T_{\text {now }} M_{G}^{n+2}} \Omega_{R} h^{2} \\
& =\left[\frac{c_{\xi}}{3 \xi(3) / 2}\right]^{2}\left[\frac{10}{g_{*}\left(T_{R H}\right)}\right]^{3 / 2} \frac{M_{X} T_{R H}^{n+1}}{M_{G}^{n+2}} \frac{4 \times 10^{24}}{(6-n)} .
\end{aligned}
$$

The result in Eq. (52) is valid as long as the $X$ particles do not reach equilibrium at temperatures larger than $T_{R H}$, or $n_{X}\left(T_{R H}\right)<n_{X}^{e q}\left(T_{R H}\right)$. This implies

$$
M_{G}>\left[\frac{8 c_{\xi} M_{P l} T_{R H}^{n+1}}{\sqrt{5}(6-n) \pi^{7 / 2} g_{*}^{1 / 2}\left(T_{R H}\right)}\right]^{1 / n+2} .
$$

This condition holds for both gravitinos and Kaluza-Klein gravitons. For a Majorana fermion with $n=2$, it requires

$$
M_{G}>\left[\frac{10}{g_{*}\left(T_{R H}\right)}\right]^{1 / 8}\left(\frac{T_{R H}}{\mathrm{GeV}}\right)^{3 / 4} 18 \mathrm{TeV} .
$$

If the condition (53) is not satisfied, then the $X$ particle density thermalizes. However, in this case, they do not freeze out before reheating. Indeed, let us consider the ratio of the interaction rate versus the expansion rate at the temperature $T$

$$
\frac{n_{X}^{e q}\langle\sigma v\rangle}{H}=\frac{3 c_{\xi} g_{*}^{1 / 2}\left(T_{R H}\right)}{\sqrt{5} \pi^{7 / 2} g_{*}(T)} \frac{M_{P I} T_{R H}^{2} T^{n-1}}{M_{G}^{n+2}} .
$$

By requiring that the condition (53) does not hold and that $T>T_{R H}$, we find

$$
\frac{n_{X}^{e q}\langle\sigma v\rangle}{H}>\frac{3(6-n)}{8} .
$$

Since for $n<6$ the right-hand side is of order unity, Eq. (56) shows that if a relativistic $X$ reaches thermal equilibrium, then it does not freeze out before reheating.

In conclusion, for relativistic particles the relevant processes determining their relic abundances occur at reheating or afterwards. This, in particular, is true for gravitinos and for the graviton Kaluza-Klein excitations with $\delta<6$ for which the cosmological bound derived in Ref. [3] applies.

\section{E. Summary of the different cases}

Because of all the various cases encountered, it is probably useful to summarize the different possibilities. Let us consider a stable weakly-interacting particle $X$ with mass $M_{X}$ and dominant $s$-wave annihilation.

When $M_{X} \gtrsim 17 T_{\mathrm{MAX}} / 4$ we are in the deep nonrelativistic regime, and the $X$ relic abundance is strongly suppressed by an exponential factor. For $17 T_{\mathrm{MAX}} / 4 \gtrsim M_{X} \gtrsim T_{\mathrm{MAX}}$ we are in the nonrelativistic case. Depending on the value of the annihilation cross section, $X$ may or may not reach an equilibrium distribution before freezing out. In the first case $\left(\alpha_{s}\right.$ $\left.>\bar{\alpha}_{s}\right), \Omega_{X}$ is given by Eq. (45), and in the second one ( $\alpha_{s}$ $<\bar{\alpha}_{s}$ ), it is given by Eq. (36). The agreement between analytic estimates and numerical integration of the Boltzmann equations is illustrated in Fig. 5.

For $T_{\mathrm{MAX}} \gtrsim M_{X} \gtrsim x_{F} T_{R H}$, where $x_{F}$ is given in Eq. (42), the particle $X$ is first relativistic, then becomes nonrelativistic and finally decouples before reheating. The relic abundance is again given by Eq. (45).

Lighter $X$ particles thermalize after reheating, erasing any previous information on their number density. The relic abundance has the ordinary expression given in Eq. (46).

We have also discussed in Sec. III D the case of very light particles with annihilation cross section suppressed by a 


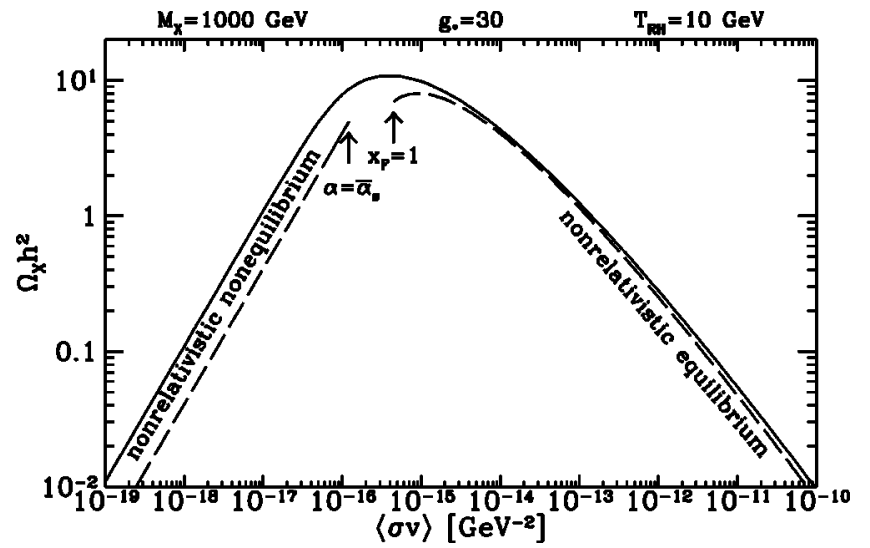

FIG. 5. Comparison of numerical vs analytic results. The nonequilibrium calculation is relevant for $\alpha<\bar{\alpha}$, shown in the figure. The equilibrium calculation assumes decoupling while nonrelativistic, or $x_{F}>1$.

heavy mass scale. In all cases of interest, the relic abundance is determined by the physics at $T_{R H}$.

\section{APPLICATIONS TO DARK MATTER CANDIDATES}

\section{A. Supersymmetry}

The neutralino is the most natural cold dark matter candidate in the context of supersymmetric extensions of the standard model. If the neutralino is dominantly a Higgsino its relic density is typically small, because of the efficient coannihilation with other neutralinos and charginos which turn out to be almost degenerate in mass. Upcoming CERN $e^{+} e^{-}$ collider LEP2 runs will be able to probe the small window left unexplored in which a light Higgsino could give a significant contribution to the present energy density of the universe [23]. Moreover, if the Higgsino is heavier than the gauge bosons, the annihilation channels into $W^{ \pm}$and $Z^{0}$ strongly depletes its relic abundance. Significant contributions to $\Omega$ then require a lightest supersymmetric particle (LSP) heavier than about $500 \mathrm{GeV}$, weakening the motivations for low-energy supersymmetry.

The case of a mainly $B$-ino lightest neutralino is much more promising for dark matter. First of all, we should recall that most of the supersymmetric models obtained from supergravity usually predict that the Higgs mixing parameter $\mu$ is large. This is because $\mu$ should compensate the large radiative corrections to the Higgs mass parameters in order to achieve the correct size of electroweak symmetry breaking. Therefore, a common expectation is that the lightest neutralino is an almost pure $B$-ino.

In the early universe, the $B$-ino will mainly annihilate into fermion pairs through $t$-channel exchange of squarks and sleptons. Exceptions occur only for pathological situations in which there is a resonant $s$-channel exchange of $Z^{0}$ or a Higgs boson. Actually, because of the large hypercharge of the right-handed electron and the expected lightness of sleptons compared to squarks, it is often a good approximation to include in the annihilation cross section only the exchange of the right-handed sleptons. Summing over three slepton de- generate families with mass $\tilde{m}_{l_{R}}$, the $B$-ino annihilation cross section parameters are

$$
\alpha_{s}=0, \quad \alpha_{p}=\frac{24 \pi \alpha^{2}}{\cos ^{4} \theta_{W}}\left(1+\frac{\tilde{m}_{l_{R}}^{2}}{M_{B}^{2}}\right)^{-2} .
$$

Notice that the annihilation process is $p$-wave suppressed because of the Majorana nature of the neutralino.

Cosmological considerations give an upper bound to the $B$-ino mass. Indeed, the requirement that charged particles are not the LSP implies $\tilde{m}_{l_{R}}>M_{B}$. The minimum allowed $B$-ino relic abundance corresponds to the maximum annihilation cross section and therefore to the minimum $\tilde{m}_{l_{R}}$. Setting $\tilde{m}_{l_{R}}=M_{B}$ in the expression for $\Omega$, one obtains an upper bound on the $B$-ino mass of about $300 \mathrm{GeV}$ (for $\Omega h^{2}$ $<0.3$ ) [13]. The bound can be weakened in the presence of resonant $s$-channel annihilations, once a small Higgsino admixture is introduced. In constrained models in which the supersymmetry-breaking masses satisfy simple universal relations at the GUT scale, this bound reduces to $200 \mathrm{GeV}$ [14].

However, as emphasized in Ref. [15], whenever the sleptons and the $B$-ino become degenerate in mass within about 10-20\%, one cannot ignore the effects of coannihilation. These effects can modify significantly the $B$-ino relic abundance, because annihilation channels involving the charged sleptons have large cross sections which are not $p$-wave suppressed. Indeed, even in the case of the constrained model, the previous limit on the $B$-ino mass can be relaxed to about $600 \mathrm{GeV}$ [15].

Coannihilation effects do not significantly modify the bound on the slepton mass for a fixed value of $M_{B}$ (as long as it is not too close to $\left.\tilde{m}_{l_{R}}\right)$. On the other hand, these bounds can rapidly disappear if we consider low values of the reheat temperature. The effect is illustrated in Fig. 6, which shows the values of slepton and $B$-ino masses incompatible with the constraint $\Omega h^{2}<1$, for different choices of $T_{R H}$, in the case of a $100 \%$ pure $B$-ino LSP. The upper bounds on $\tilde{m}_{l_{R}}$ are drastically relaxed. ${ }^{5}$ Therefore, this shows that cosmological arguments based on relic abundances, used to set upper bounds on supersymmetric particles rely on specific untested assumptions. A low reheat temperature can completely change the picture.

The same arguments can be applied to other supersymmetric dark matter candidates. One possibility which one encounters in theories with gauge-mediated supersymmetry

\footnotetext{
${ }^{5}$ This is particularly welcome in those scenarios where the supersymmetric flavor and $C P$ problems are avoided if the first two generations of sfermions are heavier than a few $\mathrm{TeV}$ and approximately degenerate in mass. If the lightest supersymmetric particle is essentially $B$-ino-like then requiring that all flavor changing neutral current and $C P$-violating processes are adequately suppressed imposes a lower limit on the $B$-ino mass of typically $200-300 \mathrm{GeV}$ [24].
} 


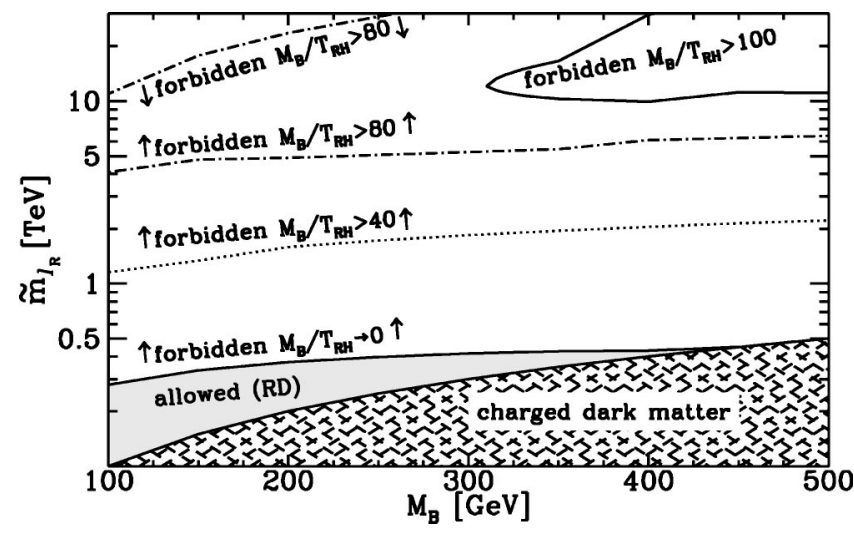

FIG. 6. The allowed region in the $M_{B}-\tilde{m}_{l_{R}}$ plane in the standard cosmology where freeze out occurs in the radiation-dominated era is indicated by the shaded region above the disallowed region where the LSP is charged and below the curve marked "forbidden $M_{B} / T_{R H} \rightarrow 0$ " above which $\Omega_{B} h^{2}>1$. Coannihilation effects, here neglected, modify the bounds in a narrow region where $B$ and $l_{R}$ are nearly mass-degenerate. If the $B$-ino freezes out during reheating the forbidden region where $\Omega_{B} h^{2}>1$ is a banana-shaped region. The allowed region of parameter space is above the charged-darkmatter region and outside the banana-shaped region. The size and location of the banana-shaped disallowed region depends on $M_{B} / T_{R H}$. Some examples are shown in the figure.

breaking $[25,26]$ is given by the messenger scalar particle with the same gauge quantum numbers of the neutrino. The cosmological upper bound on this particle mass is about 3 $\mathrm{TeV}$, much lower than the natural theoretical expectation. A low reheat temperature easily relaxes the bound.

The same can be said about the limits on unstable particles whose decays into energetic products may jeopardize the successful predictions of nucleosynthesis [18]. It is clear that our results relax the bounds in the $\left(M_{X}, \tau_{X}\right)$ plane, where $\tau_{X}$ is the decay lifetime of the unstable particle.

\section{B. Massive neutrinos}

Let us now analyze the implications of our approach for massive neutrinos, in the case in which they are stable. First, let us briefly recall the standard (i.e., when the reheat temperature is very large) prediction for the abundance of massive neutrinos. Neutrinos are initially kept in equilibrium by weak interactions. For neutrinos lighter than about $1 \mathrm{MeV}$, freeze out occurs at $T_{D} \simeq 2.3 \mathrm{MeV}$ for electron neutrinos and $T_{D} \simeq 3.7 \mathrm{MeV}$ for muon and tau neutrinos, so the neutrinos are relativistic at freeze out. The current abundance of a generic relativistic particle can be easily estimated to be [8]

$$
\Omega_{X} h^{2} \simeq \frac{g_{X}}{2} \frac{10.75}{g_{*}\left(T_{D}\right)} \frac{m_{X}}{94 \mathrm{eV}} .
$$

Here $g_{X}$ is the number of degrees of freedom of the species. Standard model (SM) relativistic neutrinos decouple from chemical equilibrium when $g_{*}\left(T_{D}\right) \simeq 10.75$. One usually concludes that the mass of SM neutrinos cannot be larger than $94 h^{2} \mathrm{eV}$, the Cowsik-McClelland bound [16].

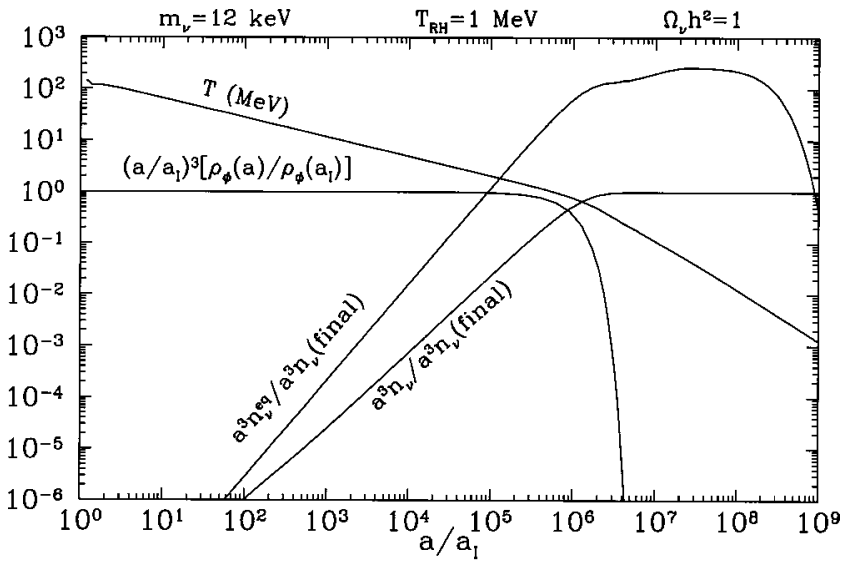

FIG. 7. The evolution of the number density per comoving volume of Dirac neutrinos for $m_{\nu}=12 \mathrm{keV}$ and $T_{R H}=1 \mathrm{MeV}$. This figure illustrates the fact that neutrinos never attain chemical equilibrium.

If neutrino masses are larger than the freeze-out temperature, they decouple from the thermal bath when they are nonrelativistic. In this case the annihilation cross section is proportional to $G_{F}^{2} m_{\nu}^{2}$, and requiring $\Omega_{\nu} h^{2} \lesssim 1$ provides a lower bound on $m_{\nu}$ of about $2 \mathrm{GeV}$, the Lee-Weinberg bound [17]. This means that neutrino masses in the range 94 $\mathrm{eV} \lesssim m_{\nu} \lesssim 2 \mathrm{GeV}$ are cosmologically ruled out. This is the celebrated Cowsik-McClelland-Lee-Weinberg (CMLW) bound $[16,17]$.

This picture has to be modified in the case in which the reheat temperature is small. The standard CMLW bound is based on the assumption that neutrinos have reached thermal and chemical equilibrium in the radiation-dominated universe. This is equivalent to the hypothesis that the maximum temperature obtained during the (last) radiation-dominated era, that is, the reheat temperature $T_{R H}$, is much larger than the decoupling temperature $T_{D}$. We have no physical evidence of the radiation-dominated era before the epoch of nucleosynthesis (i.e., temperatures above about $1 \mathrm{MeV}$ ). Therefore, let us explore the possibility that the largest temperature of the Universe during the radiation-dominated phase is very small. Indeed, it has been recently shown that the smallest value not excluded by nucleosynthesis at more than $95 \%$ C.L. is $T_{R H} \simeq 0.7 \mathrm{MeV}$ [27].

Since neutrinos have only weak interactions, it is very difficult for the thermal scatterings of particles during the reheat stage to generate SM neutrinos through processes like $e^{+} e^{-} \rightarrow \nu \bar{\nu}$ and to bring neutrinos into chemical equilibrium. Furthermore, decreasing the reheat temperature increases the rate of the expansion of the Universe, as explicitly seen in Eq. (20), making it more difficult for the weak interactions to bring the neutrinos into chemical equilibrium. Therefore, if the reheat temperature is small enough, one should expect that the SM neutrinos produced during the reheat stage never reach chemical equilibrium. In other words, at the beginning of the radiation-dominated phase neutrinos populate the thermal bath, but they have a number density, $n_{\nu}$, which is smaller than the equilibrium number density, $n_{\nu}^{e q}$. This is well illustrated in Fig. 7, which shows the evolution of the 

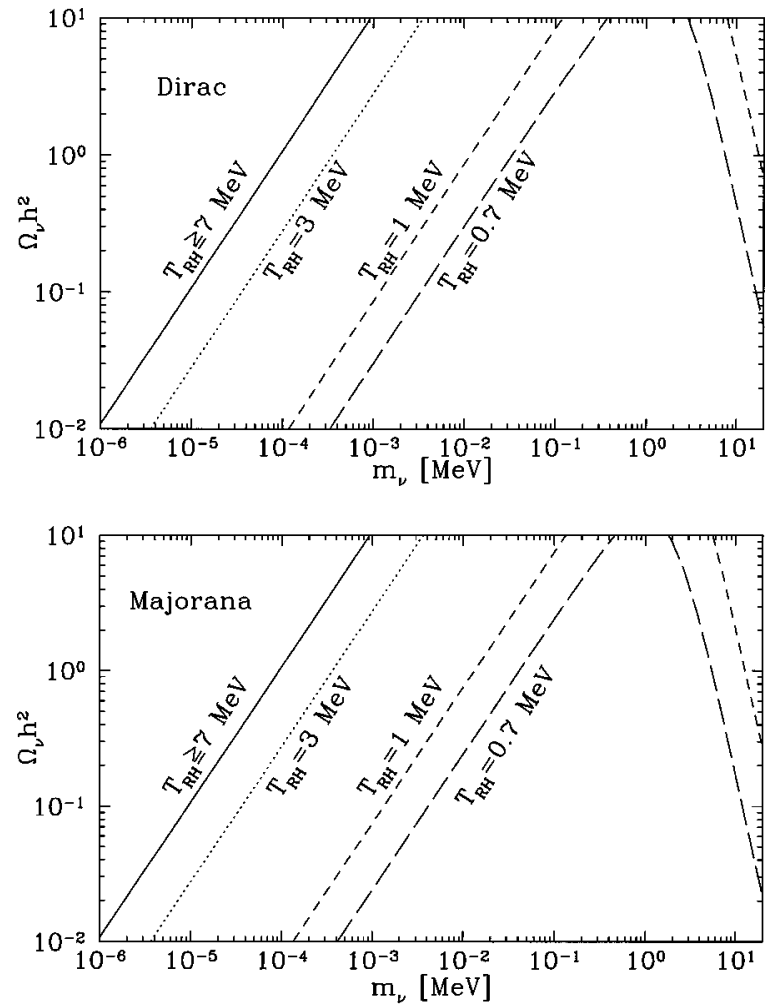

FIG. 8. The contribution to the closure density of Dirac and Majorana neutrinos for different values of the reheat temperature $\left(g_{*}=10.75\right.$ is used in the definition of $\left.T_{R H}\right)$.

number density of a muon or tau Dirac neutrino with mass $12 \mathrm{keV}$, for $T_{R H}=1 \mathrm{MeV}$. It is clear that the number densityonly grows, and it is always too small to catch up with the equilibrium number density, i.e., $n_{\nu} \ll n_{\nu}^{e q}$. This implies that neutrino annihilations are not efficient.

This result applies both to relativistic and nonrelativistic SM neutrinos, and implies that the present abundance of neutrinos in low $T_{R H}$ models is much smaller than predicted assuming that the largest temperature of the radiationdominated universe was much larger than a few MeV. This is the reason why the CMLW bound on neutrino masses is significantly relaxed in low $T_{R H}$ models.

In Fig. 8 we present a full numerical computation of the abundance of massive neutrinos in terms of $\Omega_{\nu} h^{2}$ as a function of the mass of the neutrino, for different values of the reheat temperature. In all our results we have numerically solved the Boltzmann equation making use of the exact definition of the thermally averaged cross section [28]

$$
\begin{aligned}
\langle\sigma v\rangle= & \frac{1}{4 m_{\nu}^{2} T K_{2}^{2}\left(m_{\nu} / T\right)} \int_{4 m_{\nu}^{2}}^{\infty} d s \sigma v E_{\nu} E_{\nu}^{-} \\
& \times \sqrt{s-4 m_{\nu}^{2}} K_{1}(\sqrt{s} / T),
\end{aligned}
$$

where $s$ is the center-of-mass energy, $K_{i}$ are the modified
Bessel functions and we borrowed the expressions for $\sigma v E_{\nu} E_{\nu}^{-}$from the appendix of Ref. [29]. ${ }^{6}$

For a Dirac neutrino, the treatment of the number of degrees of freedom requires some attention. In the relativistic case, only two of the four degrees of freedom are produced because the generation of the wrong-helicity states is suppressed by $\left(m_{\nu} / 2 E_{\nu}\right)^{2}$. On the other hand, in the nonrelativistic regime, all four degrees of freedom interact with full strength. Here, we make the "helicity approximation" and assume two degrees of freedom for a relativistic neutrino species and four for a nonrelativistic neutrino species.

At low reheat temperatures neutrinos of a given family $\nu_{\alpha}$ can be produced by the processes $e^{+} e^{-} \rightarrow \nu_{\alpha} \bar{\nu}_{\alpha}$ and $\nu_{\beta} \bar{\nu}_{\beta}$ $\rightarrow \nu_{\alpha} \bar{\nu}_{\alpha}$, where $\beta$ is different from $\alpha$. The inclusion of the $e^{+} e^{-}$scattering in the integrated Boltzmann equation for the number density of neutrinos $n_{\nu_{\alpha}}$ is straightforward because $e^{-}$and $e^{+}$are kept in chemical equilibrium by the fast electromagnetic processes and Eq. (3) applies. However, the inclusion of the $\nu_{\beta} \bar{\nu}_{\beta}$ scatterings is more delicate because $\nu_{\beta}$ and $\bar{\nu}_{\beta}$ are themselves not in chemical equilibrium. A complete solution of the problem would require a detailed kinetic treatment of all the neutrino distribution functions $f_{\nu}(\mathbf{p}, t)$ in momentum space. This computation is now in progress. In this paper we have limited ourselves to include the $\nu_{\beta} \bar{\nu}_{\beta}$ scatterings by defining in Eq. (59) an effective cross section $\sigma_{e f f} \equiv \sigma_{e} e^{-}+\Sigma_{\beta}\left(n_{\nu_{\beta}} / n_{\nu_{\beta}}^{e q}\right)^{2} \sigma_{\nu_{\beta} \bar{\nu}_{\beta}}$. To convince oneself of the validity of this approximation, one may notice that we recover the usual standard CMLW bound when the reheat temperature is larger than about $7 \mathrm{MeV}$. This is in agreement with the results obtained in Ref. [27] where the Boltzmann equations in momentum space were numerically solved for massless neutrinos and it was shown that for $T_{R H} \lesssim 7 \mathrm{MeV}$ the effective number of massless neutrinos $N_{\nu} \equiv \rho_{\nu} / \rho_{\nu}^{e q}$ starts deviating from 3 .

From Fig. 8 we infer that in the case in which neutrinos are relativistic, $\Omega_{\nu} h^{2}$ is approximately given by

$$
\begin{aligned}
\Omega_{\nu_{\mu}} h^{2}= & \Omega_{\nu_{\tau}} h^{2} \simeq\left(\frac{m_{\nu}}{12 \mathrm{keV}}\right)\left(\frac{T_{R H}}{\mathrm{MeV}}\right)^{3} \\
& \text { relativistic Majorana and Dirac. }
\end{aligned}
$$

The full numerical calculation shows that neutrino masses as large as $m_{\nu} \simeq 33 \mathrm{keV}$ are compatible with $\Omega_{\nu} h^{2}<1$ for the limiting reheat temperature $T_{R H}=0.7 \mathrm{MeV}$. This shows that SM neutrinos with masses up to about $33 \mathrm{keV}$ are perfectly compatible with cosmology and may even play the role of warm dark matter.

Let us now briefly see what our findings are when the neutrinos are heavier than an $\mathrm{MeV}$. This is possible only for the tau neutrino, for which the present experimental upper limit on the mass is $18.2 \mathrm{MeV}$ [30]. For $m_{\nu_{\tau}} \sim 10 \mathrm{MeV}$ the ordinary calculation of the relic abundance in a radiation-

\footnotetext{
${ }^{6}$ In Ref. [29], for the Majorana case, $C_{V}$ and $C_{A}$ have to be interchanged. We thank G. Raffelt for communications about this point.
} 
dominated universe predicts a value of $\Omega_{\nu_{\tau}} \sim 10^{4}$, which is definitely excluded. Our findings indicate that

$\left(\Omega_{\nu_{\tau}} h^{2}\right)=\left(\frac{T_{R H}}{\mathrm{MeV}}\right)^{7}\left(\frac{14 \mathrm{MeV}}{m_{\nu_{\tau}}}\right)^{3}$ nonrelativistic Dirac,
$\left(\Omega_{\nu_{\tau}} h^{2}\right)=\left(\frac{T_{R H}}{\mathrm{MeV}}\right)^{7}\left(\frac{13 \mathrm{MeV}}{m_{\nu_{\tau}}}\right)^{3}$ nonrelativistic Majorana.

The strong dependence upon the reheat temperature is easily understood by realizing that for $T_{R H}$ in the $\mathrm{MeV}$ range we are in the condition of Sec. III B $\left(\alpha_{s}<\bar{\alpha}_{s}\right.$ and $\left.\alpha_{p}<\bar{\alpha}_{p}\right)$, and therefore the neutrino relic abundance is given approximately by Eq. (36). Our numerical result is very well reproduced analytically by taking Eqs. (27) and (36) and using, for a Dirac neutrino,

$$
\alpha_{s}=\frac{G_{F}^{2} m_{\nu_{\tau}}^{4}}{2 \pi}\left(\frac{1}{2}-2 \sin ^{2} \theta_{W}+4 \sin ^{4} \theta_{W}\right),
$$

and, for a Majorana neutrino,

$$
\alpha_{p}=\frac{2 G_{F}^{2} m_{\nu_{\tau}}^{4}}{\pi}\left(\frac{1}{2}-2 \sin ^{2} \theta_{W}+4 \sin ^{4} \theta_{W}\right) .
$$

These values are obtained taking into account only the process $e^{+} e^{-} \rightarrow \nu_{\tau} \bar{\nu}_{\tau}$ in $\sigma_{e f f}$ because, as we have numerically checked, the contribution to the production of heavy tauneutrinos from light neutrino annihilations is negligible at the production time, see Eqs. (29) and (30).

It is interesting to notice that there exists a small window of $m_{\nu_{\tau}}$ and $T_{R H}$ for which the ordinary tau neutrino is an acceptable candidate for cold dark matter. However, because of the large powers of $m_{\nu_{\tau}}$ and especially of $T_{R H}$ in Eq. (61), the allowed window is very limited. Of course in this scenario there must be a conserved quantum number to keep the tau neutrino stable in order to be the dark matter; this hypothesis is in apparent contradiction with the experimental evidence on neutrino oscillations.

It has also been proposed that the tau neutrino could be a cold dark matter candidate if it had a magnetic moment of the order of $10^{-6}$ Bohr magnetons [31]. The present experimental limit on the $\nu_{\tau}$ magnetic moment of $5.4 \times 10^{-7} \mu_{B}$ rules out this possibility. We find that choosing $T_{R H}$ as low as $1 \mathrm{MeV}$ can rescue this possibility, since $\Omega_{\nu_{\tau}} h^{2} \simeq 1$ for a tau neutrino magnetic moment with the maximum allowed value.

We conclude that the cosmologically disallowed region for neutrino masses is

$$
33 \mathrm{keV} \lesssim m_{\nu} \lesssim 6 \mathrm{MeV}
$$

for a Dirac neutrino and

$$
33 \mathrm{keV} \lesssim m_{\nu} \lesssim 5 \mathrm{MeV}
$$

for a Majorana neutrino. We stress that so long as the reheat temperature is an unknown parameter, this should be considered the real CMLW bound on neutrino masses. Our findings indicate that neutrinos can still play the role of warm or cold dark matter and that the impact of massive neutrinos on nucleosynthesis has to be revisited. These and other issues are currently under investigation. Finally, we note that the above values were found assuming Maxwell-Boltzmann statistics and assuming the annihilation products are in equilibrium.

\section{Cosmological bound on axions}

The invisible axion is still the most elegant solution to the strong $C P$ problem [32,33]. An axion model has one basic free parameter, the axion mass $m_{a}$, or equivalently, the Peccei-Quinn (PQ) symmetry breaking scale $f_{P Q}$. The mass and symmetry breaking scale are related by

$$
m_{a} \simeq 0.62 \mathrm{eV} \frac{10^{7} \mathrm{GeV}}{f_{P Q} / N},
$$

where $N$ is the color anomaly of the PQ symmetry.

Several astrophysical lower limits on $f_{P Q}$ are based on the requirement that the axionic energy losses from stars, notably red-giant stars, globular-cluster stars, or the core of supernova 1987A, are not in conflict with the observed properties of these objects [34]. These limits imply $m_{a} \lesssim 10^{-2} \mathrm{eV}$ (equivalently $f_{P Q} \gtrsim 10^{9} \mathrm{GeV}$ ), indicating that axions, if they exist, are both extremely light and very weakly interacting.

An upper bound on the PQ scale comes from cosmological considerations. Let us call $\Theta$ the strong $C P$-violating phase. Today $\Theta$ is anchored at the $C P$-conserving value, $\Theta=0$. However, the axion mass is very temperaturedependent [35]

$$
m_{a}(T) \simeq 0.1 \quad m_{a}\left(\frac{\Lambda_{Q C D}}{T}\right)^{3.7},
$$

where $\Lambda_{Q C D} \simeq 200 \mathrm{MeV}$ is the QCD scale and the relation is valid for $T \gg \Lambda_{Q C D} / \pi$. At very high temperatures the axion is essentially massless. This means that no special value of $\Theta$ is specified by the dynamics, and all values of the phase are equivalent. The axion mass turns on at a temperature $T_{1}$ such that $m_{a}\left(T_{1}\right) \simeq 3 H\left(T_{1}\right)$, and the axion field starts evolving toward the minimum at $\Theta=0$, eventually oscillating around it. These cosmic oscillations of the axion field correspond to a zero-momentum condensate of axions which does not decay. The energy density in axions today from this misalignment mechanism exceeds the critical density unless $f_{P Q}$ $\lesssim 10^{12} \mathrm{GeV}$ [36]. This result has always been considered particularly disappointing from the theoretical point of view, since weakly coupled string theory possesses numerous axion candidates, whose decay constant is, however, of the order of the string scale and therefore much larger than $10^{12}$ $\mathrm{GeV}$.

The purpose of the present section is to demonstrate that the cosmological bound on the PQ scale is significantly relaxed if we make the assumption that the reheat temperature 
is smaller than the QCD scale (similar considerations have also been made in Refs. [37,38]).

Suppose then that $T_{R H} \leq \Lambda_{Q C D} \leq T_{\mathrm{MAX}}$. This means that the axion coherent oscillations commence when the universe is still matter-dominated and reheating is not completed. During this epoch the Hubble rate is given by Eq. (20), and the axion mass is still given by Eq. (67) since the universe is populated by a thermal bath between $T_{\mathrm{MAX}}$ and $T_{R H}$. Axions start oscillating at a temperature $T_{1}$ when $m_{a}\left(T_{1}\right)$ $\simeq 3 H\left(T_{1}\right)$ :

$$
\begin{aligned}
T_{1} \simeq & \left(\frac{m_{a}}{10^{-5} \mathrm{eV}}\right)^{1 / 7.7}\left[\frac{g_{*}\left(T_{R H}\right)}{10}\right]^{1 / 15.4}\left[\frac{g_{*}\left(T_{1}\right)}{10}\right]^{-1 / 7.7} \\
& \times\left(\frac{T_{R H}}{1 \mathrm{MeV}}\right)^{2 / 7.7} 160 \mathrm{MeV} .
\end{aligned}
$$

At temperatures $T \leqq T_{1}$ the number density of axions scales like $a^{-3}$ even though the axion mass is still varying. At the reheat temperature we have

$$
\begin{aligned}
n_{a}\left(T_{R H}\right) & =n_{a}\left(T_{1}\right)\left[\frac{a\left(T_{1}\right)}{a\left(T_{R H}\right)}\right]^{3} \\
& =\frac{\sqrt{5 \pi^{3}} \bar{\Theta}_{1}^{2}}{2}\left(\frac{f_{P Q}}{N}\right)^{2} \frac{g_{*}^{3 / 2}\left(T_{R H}\right)}{g_{*}\left(T_{1}\right)} \frac{T_{R H}^{6}}{T_{1}^{4} M_{P l}},
\end{aligned}
$$

where $\bar{\Theta}_{1}$ is the initial displacement of the $C P$ phase. Notice, in particular, that the ratio $n_{a} / s$ does not remain constant during the cosmological evolution from the temperature $T_{1}$ to $T_{R H}$. This is because there is a continuous release of entropy. However, when reheating is completed and the universe enters a radiation-dominated phase, the ratio $n_{a} / s$ is conserved, and one can easily compute the present abundance of axions from the misalignment mechanism

$$
\begin{aligned}
\Omega_{a} h^{2}= & \frac{m_{a} n_{a}\left(T_{R H}\right)}{8 \rho_{R}\left(T_{R H}\right)} \frac{T_{R H}}{T_{\text {now }}} \Omega_{R} h^{2} \\
= & 2.1 \times 10^{-7}\left(\frac{\bar{\Theta}_{1}}{\pi / \sqrt{3}}\right)^{2}\left[\frac{g_{*}\left(T_{R H}\right)}{10}\right]^{0.24} \\
& \times\left[\frac{g_{*}\left(T_{1}\right)}{10}\right]^{-0.48}\left(\frac{10^{-5} \mathrm{eV}}{m_{a}}\right)^{1.52}\left(\frac{T_{R H}}{1 \mathrm{MeV}}\right)^{1.96} .
\end{aligned}
$$

Requiring that $\Omega_{a} h^{2} \lesssim 1$ gives

$$
\begin{aligned}
\frac{f_{P Q}}{N} \lesssim 1.6 \times 10^{16}\left(\frac{T_{R H}}{1 \mathrm{MeV}}\right)^{-1.3} \mathrm{GeV} \\
\left(T_{R H} \lesssim \Lambda_{Q C D} \lesssim T_{\mathrm{MAX}}\right) .
\end{aligned}
$$

Therefore, the cosmological axion problem is ameliorated. ${ }^{7}$ Furthermore, in the strong-coupling vacuum described in Ref. [39], the QCD axion might be a boundary modulus. Dimensional analysis suggests approximately $10^{16} \mathrm{GeV}$ for the decay constant of such a boundary axion, not necessarily in contradiction with the upper bound of Eq. (71), see also the discussion in Ref. [40].

\section{IMPLICATIONS FOR GUT BARYOGENESIS AND LEPTOGENESIS}

The explanation of the observed baryon asymmetry $(B)$ in the early universe, of the order of $10^{-11}$ in units of the entropy density, remains a fundamental cosmological question [41]. Several theories with typical energy scale much higher than the electroweak scale can explain the observed baryon asymmetry. For instance, in grand unified theories (GUT's) the out-of-equilibrium decay of heavy Higgs particles may be responsible for the direct generation of the baryon asymmetry [42]. Alternatively, the baryon asymmetry may be produced from a lepton asymmetry $(L)$ [43] using the fact that any lepton asymmetry is reprocessed into a baryon number by the anomalous sphaleron transitions [44]. In the simplest scenario, the lepton asymmetry is generated by the out-ofequilibrium decay of a massive right-handed Majorana neutrino, whose addition to the standard model spectrum breaks $B-L$.

However, any scenario for the generation of the baryon asymmetry based on the out-of equilibrium decay of some heavy particle $X$ depends crucially on the assumption that these particles were nearly as abundant as photons at very high temperatures. This imposes a lower bound on the reheat temperature, $T_{R H} \geq M_{X}$. On the other hand, in supersymmetric models the requirement that not too many gravitinos are thermally produced after inflation provides a stringent upper bound on the reheat temperature of about $10^{8}-10^{10} \mathrm{GeV}[5]$. If this bound is violated, the decay products of the gravitino destroy light nuclei by photodissociation and hadronic showers, thus ruining the successful predictions of nucleosynthesis. Therefore, any out-of-equilibrium decay scenario would require $M_{X} \lesssim 10^{8}-10^{10} \mathrm{GeV}$, a condition which looks particularly problematic for GUT-inspired baryogenesis.

In order to relax this limit one usually envisages two possibilities. Either, the heavy particles are produced directly through the inflaton perturbative decay process [45] (this requires that the mass of the inflaton is larger than $M_{X}$ ) or they are generated through nonperturbative process taking place at the preheating stage (see Ref. [46] in the case of GUT Higgs boson induced baryogenesis and Ref. [47] in the case of leptogenesis).

\footnotetext{
${ }^{7}$ In cosmologies where the universe is dominated early on by the coherent oscillations of some moduli field the axion bound is significantly weakened, as had been already observed in Refs. [37,38]. In Ref. [38], however, the estimate on the upper bound on $f_{P Q}$ did not take into account the presence of the thermal bath before the completion of reheating, and therefore neglected the dependence of the axion mass on the temperature.
} 
In this section we wish to show that the heavy particles $X$ may be abundantly produced by thermal scatterings during the reheat stage even though the reheat temperature $T_{R H}$ is smaller than $M_{X}$. Again, this is made possible by the fact that $T_{R H}$ is not the maximum temperature of the universe during reheating. For the sake of concreteness we will focus on the leptogenesis scenario, but our findings can be easily generalized to any out-of-equilibrium scenario.

Let us indicate by $N=n_{N_{1}} a^{3}$ the number density per comoving volume of the lightest right-handed neutrino, the one whose final decay (into left-handed leptons and Higgs bosons) is responsible for the generation of the lepton asymmetry. Following the notations of Sec. II A, we can write the Boltzmann equation for $N$ as

$$
\frac{d N}{d A}=-\frac{c_{N} A^{1 / 2}\left(N-N_{e q}\right)}{\sqrt{\Phi}}
$$

where

$$
c_{N} \equiv \sqrt{\frac{3}{8 \pi}} \frac{M_{P l}}{T_{R H}^{2}}\left(\Gamma_{N_{1}}+2 \Gamma_{h, s}+4 \Gamma_{h, t}\right) .
$$

Here $\Gamma_{N_{1}}$ is the decay rate of $N_{1}$ (for the processes $N_{1}$ $\left.\rightarrow H^{\dagger} l_{L}, H \bar{l}_{L}\right) ; \Gamma_{h, s}$ and $\Gamma_{h, t}$ are the rates of the scattering processes containing $N_{1}$ in the final state, mediated by the Higgs boson in the $s$ channel $\left(\bar{t}_{R} q_{L}^{(3)} \rightarrow \bar{l}_{L} N_{1}\right)$ and in the $t$ channel $\left(l_{L} q_{L}^{(3)} \rightarrow \bar{t}_{R} N_{1}\right)$, respectively.

Let us suppose first that $T_{\mathrm{MAX}} \lesssim M_{1}$, where $M_{1}$ is the $N_{1}$ mass. Under this assumption we have

$$
\Gamma_{N_{1}}=\frac{\lambda_{1}^{2}}{8 \pi} M_{1}, \quad \Gamma_{h, s}=\frac{3 \lambda_{1}^{2} \lambda_{t}^{2}}{32 \pi^{5}} \frac{T^{3}}{M_{1}^{2}}, \quad \Gamma_{h, t}=\frac{3 \lambda_{1}^{2} \lambda_{t}^{2}}{32 \pi^{3}} T \ln \frac{M_{1}}{m_{h}},
$$

where $\lambda_{1}^{2} \equiv\left(\lambda \lambda^{\dagger}\right)_{11}$, with $\lambda_{i j}$ Yukawa coupling of $N_{1}$, and $\lambda_{t}$ is the top Yukawa coupling. For a more transparent physical interpretation, it is convenient to express $\lambda_{1}^{2}$ in terms of the parameter

$$
m_{1} \equiv \frac{\lambda_{1}^{2}}{2 \sqrt{2} G_{F} M_{1}} .
$$

In the limit of small mixing angles the parameter $m_{1}$ coincides with the mass of one of the light (mainly left-handed) neutrinos.

If the right-handed neutrinos do not reach an equilibrium density $\left(N \ll N_{e q}\right)$, we can approximate Eq. (72) by

$$
\frac{d N}{d A}=\frac{c_{N} A^{1 / 2} N_{e q}}{\sqrt{\Phi_{I}}}
$$

Along the same lines of Sec. III A, we can integrate Eq. (76) by approximating it to a Gaussian integral in the full range between $A=1$ and $A=\infty$. This is a good approximation because the exponential suppression in $N_{e q}$ makes the right- hand side of Eq. (76) negligible anywhere outside a small interval of scale factors centered around $A=A_{*}$ corresponding to $T_{*}=M_{1} / 10$ for the inverse decay, and to $T_{*}=M_{1} / 9$ for the Higgs-mediated $\Delta L=1$ processes. It is easy to show that the main source of right-handed neutrinos is represented by the inverse decays whose contribution to $N_{\infty}$ is given by

$$
N_{\infty} \simeq \frac{c}{g_{*}^{3 / 2}} \frac{\Gamma_{N_{1}} M_{P l}^{3} H_{I}^{2} T_{R H}^{3}}{M_{1}^{9}}
$$

where

$$
c=\frac{72 e^{-10} 10^{9}}{\sqrt{5} \pi^{11 / 2}}=2.7 \times 10^{3} .
$$

Notice that the final abundance is suppressed only by powers of the right-handed mass, there is no Boltzmann suppression $\exp \left(-M_{1} / T_{R H}\right)$. Furthermore, the abundance is proportional to the rate of production (accumulation) $\Gamma_{N_{1}}$. However, the consistency condition $N_{\infty} \lesssim N_{e q}\left(T_{*}\right)$ gives an upper bound on $\Gamma_{N_{1}}$

$$
\Gamma_{N_{1}} \lesssim \frac{\pi}{8000} g_{*}^{1 / 2} \frac{M_{1}^{4}}{M_{P l} T_{R H}^{2}},
$$

or, equivalently, an upper bound on $m_{1}$

$$
m_{1}<\left(\frac{g_{*}}{100}\right)^{1 / 2}\left(\frac{M_{1}}{T_{R H}}\right)^{2} 2.5 \times 10^{-7} \mathrm{eV} .
$$

These bounds can also be expressed in terms of a more familiar quantity

$$
\left.K_{*} \equiv \frac{\Gamma_{N_{1}}}{H}\right|_{T=T_{*}} \lesssim 1
$$

This condition assures that when the right-handed neutrinos are produced, their direct decay is inefficient. The limiting case $K_{*} \sim 1$ would mean that the right-handed neutrinos enter into chemical equilibrium as soon as they are generated.

The right-handed neutrinos may decay before or after the universe reaches the reheat temperature $T_{R H}$, depending on the value of $\lambda_{1}$. Suppose that they decay after the end of the reheat stage (which is, for instance, always the case when $\left.M_{1} \lesssim 8 T_{R H}\right)$. This means that at $T=T_{R H}$, the ratio of the number density of right-handed neutrinos to the entropy density is given by

$$
\frac{n_{N_{1}}}{s\left(T_{R H}\right)}=\frac{25 \pi c}{g_{*}^{3 / 2}} \frac{\Gamma_{N_{1}} M_{P l} T_{R H}^{7}}{M_{1}^{9}} .
$$

This ratio remains constant until the right-handed neutrinos decay generating a lepton asymmetry

$$
L=\frac{10^{5}}{8} \frac{\epsilon}{g_{*}^{3 / 2}} \frac{\Gamma_{N_{1}} M_{P l} T_{R H}^{7}}{M_{1}^{9}},
$$


where we have indicated by $\epsilon$ the small parameter containing the information about the $C P$-violating phases and the loop factors and we have again taken into account the factor of $1 / 8$ due to the release of entropy after $T_{R H}$. The corresponding baryon asymmetry is $B=(28 / 79) L$, assuming only standard model degrees of freedom [44], and therefore

$$
B=\epsilon\left(\frac{100}{g_{*}}\right)^{3 / 2}\left(\frac{T_{R H}}{M_{1}}\right)^{7}\left(\frac{m_{1}}{10^{-7} \mathrm{eV}}\right) 7 \times 10^{-3} .
$$

By virtue of the bound of Eq. (79), this baryon asymmetry is constrained to be smaller than the critical value of $2(\epsilon)$ $\left.g_{*}\right)\left(T_{R H} / M_{1}\right)^{5}$ (and of $2 \times 10^{-5} \epsilon / g_{*}$ if we use the constraint $\left.T_{*}>T_{R H}\right)$. The requirement that $B$ is larger than 2 $\times 10^{-11}$ implies

$$
M_{1} \lesssim 16\left(\frac{100}{g_{*}}\right)^{1 / 5}\left(\frac{\epsilon}{10^{-3}}\right)^{1 / 5} T_{R H} .
$$

It is easy to convince oneself that this is also the result in the case in which the right-handed neutrinos decay before the reheat stage is over. Equation (85) provides a necessary condition on the mass $M_{1}$ of the lightest right-handed neutrino in a leptogenesis scenario, correcting the naive estimate $M_{1}$ $\lesssim T_{R H}$. The relaxation of the naive bound by more than one order of magnitude is certainly welcome to make leptogenesis more compatible with the cosmological gravitino problem.

Let us suppose now that $T_{\mathrm{MAX}} \gtrsim M_{1} \gtrsim T_{R H}$, and that inverse decays or production processes containing the $N_{1}$ in the final states can bring the right-handed neutrinos to equilibrium before they become nonrelativistic. This amounts to requiring that the $\Delta L=1$ interactions with total rate $\gamma_{N_{1}}$ $=\left(\Gamma_{N_{1}}+2 \Gamma_{h, s}+4 \Gamma_{h, t}\right)$ are in thermal equilibrium at $T$ $\gtrsim M_{1}$. Therefore the standard out-of-equilibrium parameter

$$
\left.K \equiv \frac{\gamma_{N_{1}}}{H}\right|_{T=M_{1}}
$$

is larger than unity.

In this case, the lepton asymmetry can be written as

$$
L=\frac{45}{2^{9 / 2} \pi^{7 / 2}} \frac{\epsilon z_{f}^{3 / 2} e^{-z_{f}}}{g_{*}}\left(\frac{T_{R H}}{T_{f}}\right)^{5} .
$$

Here $z_{f} \equiv M_{1} / T_{f}$, where $T_{f}$ is the temperature at which the processes that damp the baryon asymmetry go out of equilibrium, and the last factor in Eq. (87) accounts for the dilution caused by the expansion in the pre-reheat phase. We are assuming that $T_{f}$ is larger than the reheat temperature $T_{R H}$.

If the inverse decay dominates over scatterings (we will later quantify this condition), then $T_{f}$ is approximately determined by

$$
\Gamma_{I D}=\left.H\right|_{T=T_{f}},
$$

where the inverse decay rate at $T<M_{X}$ is

$$
\Gamma_{I D}=\frac{\pi^{1 / 2}}{2 \sqrt{2}} z^{3 / 2} e^{-z} \Gamma_{N_{1}}
$$

Here $z \equiv M_{1} / T$ and $\Gamma_{N_{1}}$ is given in Eq. (74). In terms of the parameter $K$, defined by

$$
\begin{aligned}
\left.K \equiv \frac{\Gamma_{N_{1}}}{H}\right|_{T=M_{1}} & =\frac{3 G_{F} m_{1} T_{R H}^{2} M_{P l}}{2 \sqrt{10} \pi^{5 / 2} g_{*}^{1 / 2} M_{1}^{2}} \\
& =\frac{m_{1}}{3 \times 10^{-3} \mathrm{eV}}\left(\frac{100}{g_{*}}\right)^{1 / 2}\left(\frac{T_{R H}}{M_{1}}\right)^{2},
\end{aligned}
$$

Eq. (88) becomes $K z_{f}^{11 / 2} e^{-z_{f}} \simeq 1$, which is approximately solved (for $K \leqq 10^{5}$ ) by $z_{f} \simeq 16 K^{0.06}$. Replacing $z_{f}$ in Eq. (87), we find

$$
L=\frac{45}{\sqrt{2} \pi^{7 / 2}}\left(\frac{T_{R H}}{M_{1}}\right)^{5} \frac{\epsilon}{g_{*} K^{0.94}} .
$$

Finally, the baryon asymmetry $B=(28 / 79) L$ is given by

$$
B \simeq \epsilon\left(\frac{100}{g_{*}}\right)^{1 / 2}\left(\frac{10^{-3} \mathrm{eV}}{m_{1}}\right)\left(\frac{T_{R H}}{M_{1}}\right)^{3} 6 \times 10^{-3} .
$$

Equation (92) is valid as long as inverse decay processes dominate over $\Delta L=2$ scattering processes in damping the baryon asymmetry. Let us now study the condition under which this hypothesis is justified. The rate for leptonviolating scatterings mediated by $N_{1}$ exchange in the $s$ or $t$ channels, for $T<M_{1}$, is given by

$$
\Gamma_{\Delta L=2}=\frac{7 G_{F}^{2} M_{1}^{3} m_{1}^{2} z^{-3}}{2 \pi^{3}} .
$$

Therefore, the $\Delta L=2$ scatterings are out-of-equilibrium at high temperatures and equilibrate at a temperature corresponding to

$$
z_{f}^{\Delta L=2}=\frac{2 \sqrt{5} \pi^{9 / 2} g_{*}^{1 / 2} M_{1}}{21 G_{F}^{2} M_{P l} m_{1}^{2} T_{R H}^{2}} .
$$

The assumption that led to Eq. (92) is then valid as long as $z_{f}^{\Delta L=2}>M_{1} / T_{R H}$, which implies

$$
m_{1}<\left(\frac{g_{*}}{100}\right)^{1 / 4}\left(\frac{10^{10} \mathrm{GeV}}{T_{R H}}\right)^{1 / 2} \mathrm{eV} .
$$

When the condition (95) is not satisfied, $\Delta L=2$ scatterings lead to an exponential suppression of the baryon asymmetry.

The baryon asymmetry in Eq. (92) is valid when three conditions are verified: the right-handed neutrinos $N_{1}$ reach equilibrium, which implies $K>1$; the temperature $T_{f}$ is larger than $T_{R H}$, which implies $M_{1}>16 T_{R H}$; Eq. (95) is satisfied. These three conditions together imply a maximum value of $M_{1}=\left(100 / g_{*}\right)^{1 / 2} 3 \times 10^{11} \mathrm{GeV}$, corresponding to a maximum $T_{R H}=\left(100 / g_{*}\right)^{1 / 2} 2 \times 10^{10} \mathrm{GeV}$. The maximum baryon asymmetry, achieved when $K \simeq 1$, is $B \simeq \epsilon(100 /$ 
$\left.g_{*}\right)\left(T_{R H} / M_{1}\right)^{5} 2 \times 10^{-3}$. Therefore, in this case, a sufficiently large baryon asymmetry requires $M_{1} \simeq 10 T_{R H}$ and $\epsilon \simeq 10^{-3}$.

\section{CONCLUSIONS}

In this paper we studied the observational consequences of having a reheat temperature $T_{R H}$ smaller that the characteristic temperature at which a certain cosmological process occurs. We first described the dynamics of reheating and derived general expressions for the relic abundance of particles whose standard freeze-out temperature is larger than $T_{R H}$. For nonrelativistic particles we found two different regimes. If the annihilation cross section $\langle\sigma v\rangle$ is smaller than the critical value in Eqs. (37),(38), than the present relic abundance is proportional $\langle\sigma v\rangle$, see Eq. (36). In the other case, the relic abundance is inversely proportional to $\langle\sigma v\rangle$, see Eq. (45), but because of the fast expansion before reheating its expression differs from the usual result in a radiationdominated universe.

We applied our general results on relic abundances in low- $T_{R H}$ cosmologies to several cases of interest. A first result is that the usual unitarity bound of $340 \mathrm{TeV}$ on stableparticle masses can be relaxed. The new excluded ranges of stable-particle masses as functions of $T_{R H}$ are shown in Fig. 4.

We revisited the parameter regions of supersymmetric models leading to viable cold dark-matter candidates in the light of low $T_{R H}$. In particular, we found that the upper bound on the slepton mass, as a function of the LSP $B$-ino mass, can be significantly relaxed, as quantitatively shown in Fig. 6. Large regions of parameter space that have been considered to be ruled out by cosmological arguments can instead give a relic neutralino density close to the critical value.

Next, we considered how a low reheat temperature can reduce the relic abundance of massive neutrinos, here assumed to be stable. The requirement that $\Omega_{\nu} h^{2} \lesssim 1$ gives the CMLW bound $m_{\nu} \lesssim 94 \mathrm{eV}$ only if $T_{R H}$ is larger than about 7 $\mathrm{MeV}$. The bound becomes significantly weaker for lower $T_{R H}$; for instance, $m_{\nu} \lesssim 12 \mathrm{keV}$ for $T_{R H}=1 \mathrm{MeV}$. For very massive neutrinos the Lee-Weinberg bound, $m_{\nu} \gtrsim 2 \mathrm{GeV}$, is also modified. Again for $T_{R H}=1 \mathrm{MeV}$, the limit becomes $m_{\nu}>14(13) \mathrm{MeV}$ for a Dirac (Majorana) neutrino. This means that there is even the possibility that a stable $\nu_{\tau}$ with mass consistent with the direct experimental limit $\left(m_{\nu_{\tau}}\right.$ $<18.2 \mathrm{MeV}$ ) freezes out when it is still nonrelativistic and becomes cold dark matter. Indeed, for $T_{R H}=0.7 \mathrm{MeV}$, which is the lowest value of $T_{R H}$ not excluded by nucleosynthesis, the constraint $\Omega_{\nu_{\tau}} h^{2} \lesssim 1$ excludes only the range of masses $33 \mathrm{keV}<m_{\nu_{\tau}}<6 \mathrm{MeV}$ (Dirac) and $33 \mathrm{keV}<m_{\nu_{\tau}}<5 \mathrm{MeV}$ (Majorana). This result resurrects the possibility of neutrinos as warm dark matter. For instance, if $T_{R H}=1 \mathrm{MeV}, \Omega_{\nu} h^{2}$ $=0.3$ for a neutrino of mass $4 \mathrm{keV}$.

The requirement that the energy stored today in the axion oscillations (caused by a misalignment between its hightemperature and low-temperature configurations) is not larger than the critical value imposes a bound on the Peccei-
Quinn symmetry breaking scale, $f_{P Q} \lesssim 10^{12} \mathrm{GeV}$. If $T_{R H}$ is less than $\Lambda_{Q C D}$, this bound can be relaxed to values close to the GUT scale.

Finally, we have investigated the impact of low $T_{R H}$ in the explanation of the observed baryon asymmetry by a leptogenesis mechanism. In this context, low $T_{R H}$ means $T_{R H}$ $<M_{1}$, where $M_{1}$ is the mass of the lightest of the three right-handed neutrinos. Therefore, we discuss here values of $T_{R H}$ much larger than in the previous cases. However, the formalism is the same, because what matters is that $T_{R H}$ is less than the relevant physical energy scale. We have found new expressions of the baryon asymmetry as functions of $T_{R H}$, see Eqs. (84) and (92). Moreover, values of $M_{1}$ an order of magnitude larger than $T_{R H}$ can still lead to a sufficient density of right-handed neutrinos to explain the baryon asymmetry. This is a welcome and important result, when one tries to make leptogenesis consistent with the cosmological gravitino problem.

\section{ACKNOWLEDGMENTS}

We have benefited from conversations with N. ArkaniHamed, R. Barbieri, M. Barnett, S. Dodelson, A. Dolgov, L. Hall, H. Murayama, G. Raffelt, R. Rattazzi, and L. Roszkowski. A.R. thanks the Theoretical Astrophysics Group at Fermilab, the Theory Group at NYU and the LBNL Theory Group for the kind hospitality during the last stages of this work. E.W.K. was supported by the Department of Energy and NASA under Grant NAG5-7092.

\section{APPENDIX: EVOLUTION AFTR $T_{R H}$}

Numerical results show that there is about a factor of 8 increase in the comoving entropy after $T_{R H}$, and that the value of $\Phi$ at $T=T_{R H}$ is $\Phi_{R H}=0.79 \Phi_{I}$, i.e., only about $21 \%$ of the comoving $\phi$ energy density has been extracted at reheat time. In this appendix we will give an explanation of these results.

Assume that at $T_{R H}$ the radiation energy density is less than the $\phi$ energy density. Then the evolution equation for $\Phi$ is

$$
\frac{d \Phi}{d A}=-\left(\frac{\pi^{2} g_{*}}{30}\right)^{1 / 2} A^{1 / 2} \Phi^{1 / 2}
$$

Integrating this equation from $A=1$ to $A=A_{R H}$ with the initial condition $\Phi=\Phi_{I}$, we obtain

$$
\left(\frac{\Phi_{R H}}{\Phi_{I}}\right)^{1 / 2}=1-\frac{1}{3}\left(\frac{\pi^{2} g_{*}}{30}\right)^{1 / 2} \Phi_{I}^{-1 / 2} A_{R H}^{3 / 2} \quad\left(A_{R H} \gg 1\right) .
$$

Evaluating Eq. (18) at $T=T_{R H}$ we obtain

$$
A_{R H}^{3}=\frac{24}{5 \pi^{2} g_{*}} \Phi_{I}
$$

Using this relation, we can estimate 


$$
\frac{\Phi_{R H}}{\Phi_{I}}=\left(\frac{13}{15}\right)^{2} \sim 0.75
$$

This is in good agreement with the numerical results and is independent of model parameters.

The second step is an estimate of how much entropy is released after $T_{R H}$, if $\Phi_{R H}=0.75 \Phi_{I}$. Let us make the crude approximation that the entropy release is instantaneous just after $T_{R H}$. Then $\Delta \rho_{R}=\Delta \rho_{\phi}$, and therefore

$$
\Delta \rho_{R}=0.75 \frac{\Phi_{I} T_{R H}^{4}}{A_{R H}^{3}} .
$$

Using Eq. (A3) we obtain

$$
\Delta \rho_{R}=0.16 \pi^{2} g_{*} T_{R H}^{4},
$$

which gives

$$
\frac{\Delta \rho_{R}}{\rho_{R}} \simeq 5 \text {. }
$$

Therefore the analytic estimate in the case of the instantaneous approximation predicts a model-independent factor of $5^{3 / 4} \simeq 3.4$ for the release of entropy after $T_{R H}$. The model dependence comes in if we relax the assumption of instantaneous release of entropy, increasing the estimate, since $\rho_{\phi}$ redshifts like $A^{-3}$. The numerical result shows an increase of about a factor of 8 in the comoving entropy after $T_{R H}$.
[1] A. H. Guth, Phys. Rev. D 23, 347 (1981); for a recent review, see D. H. Lyth and A. Riotto, Phys. Rep. 314, 1 (1999).

[2] N. Arkani-Hamed, S. Dimopoulos, and G. Dvali, Phys. Lett. B 429, 263 (1998).

[3] N. Arkani-Hamed, S. Dimopoulos, and G. Dvali, Phys. Rev. D 59, 086004 (1999).

[4] D. Lindley, Astrophys. J. 294, 1 (1985); J. Ellis et al., Nucl. Phys. B259, 175 (1985); S. Dimopoulos et al., ibid. B311, 699 (1988); J. Ellis et al., ibid. B373, 399 (1992); M. Kawasaki and T. Moroi, Prog. Theor. Phys. 93, 879 (1995).

[5] J. Ellis, A. Linde, and D. Nanopoulos, Phys. Lett. 118B, 59 (1982); D. Nanopoulos, K. Olive, and M. Srednicki, ibid. 127B, 30 (1983); J. Ellis, J. Kim, and D. Nanopoulos, ibid. 145B, 181 (1984).

[6] R. Kallosh, L. Kofman, A. D. Linde, and A. Van Proeyen, Phys. Rev. D 61, 103503 (2000); G. F. Giudice, I. I. Tkachev, and A. Riotto, J. High Energy Phys. 08, 009 (1999); G. F. Giudice, A. Riotto, and I. Tkachev, ibid. 11, 036 (1999); G. Felder, L. Kofman, and A. D. Linde, ibid. 02, 027 (2000).

[7] D. J. Chung, E. W. Kolb, and A. Riotto, Phys. Rev. D 60, 063504 (1999).

[8] E. W. Kolb and M. S. Turner, The Early Universe (AddisonWesley, Menlo Park, CA, 1990).

[9] O. Pantano and A. Riotto, Phys. Lett. B 307, 49 (1993).

[10] D. J. Chung, E. W. Kolb, and A. Riotto, Phys. Rev. D 59, 023501 (1999); Phys. Rev. Lett. 81, 4048 (1998); E. W. Kolb, D. J. Chung, and A. Riotto, hep-ph/9810361.

[11] K. Griest and M. Kamionkowski, Phys. Rev. Lett. 64, 615 (1990).

[12] G. Jungman, M. Kamionkowski, and K. Griest, Phys. Rep. 267, 195 (1996).

[13] K. A. Olive and M. Srednicki, Phys. Lett. B 230, 78 (1989).

[14] P. Nath and R. Arnowitt, Phys. Rev. Lett. 70, 3696 (1993); G. L. Kane, C. Kolda, L. Roszkowski, and J. D. Wells, Phys. Rev. D 49, 6173 (1994).

[15] J. Ellis, T. Falk, and K. A. Olive, Phys. Lett. B 444, 367 (1998); J. Ellis, T. Falk, K. A. Olive, and M. Srednicki, Astropart. Phys. 13, 181 (2000).

[16] R. Cowsik and J. McClelland, Phys. Rev. Lett. 29, 669 (1972).

[17] B. W. Lee and S. Weinberg, Phys. Rev. Lett. 39, 165 (1977).

[18] For a review, see S. Sarkar, Rep. Prog. Phys. 59, 1493 (1996).
[19] J. McDonald, Phys. Rev. D 43, 1063 (1991).

[20] J. McDonald, Phys. Rev. D 61, 083513 (2000).

[21] R. Allahverdi, Phys. Rev. D 62, 063509 (2000).

[22] T. Moroi and L. Randall, Nucl. Phys. B570, 455 (2000).

[23] J. Ellis, T. Falk, G. Ganis, K. A. Olive, and M. Schmitt, Phys. Rev. D 58, 095002 (1998).

[24] T. Gherghetta, A. Riotto, and L. Roszkowski, Phys. Lett. B 440, 287 (1998)

[25] S. Dimopoulos, G. F. Giudice, and A. Pomarol, Phys. Lett. B 389, 37 (1996).

[26] T. Han and R. Hempfling, Phys. Lett. B 415, 161 (1997).

[27] M. Kawasaki, K. Kohri, and N. Sugiyama, Phys. Rev. D 62, 023506 (2000).

[28] E. W. Kolb and S. Raby, Phys. Rev. D 27, 2990 (1983); P. Gondolo and G. Gelmini, Nucl. Phys. B360, 145 (1991).

[29] J. B. Rehm, G. G. Raffelt, and A. Weiss, Astron. Astrophys. 327, 443 (1997).

[30] Particle Data Group, C. Caso et al., Eur. Phys. J. C 3, 1 (1998).

[31] G. F. Giudice, Phys. Lett. B 251, 460 (1990).

[32] R. D. Peccei and H. R. Quinn, Phys. Rev. Lett. 38, 1440 (1977).

[33] For a recent review, see for instance, R. D. Peccei, hep-ph/9807514.

[34] For a recent review, see G. Raffelt, Nucl. Phys. B (Proc. Suppl.) 77, 456 (1999).

[35] D. J. Gross, R. D. Pisarski, and L. G. Yaffe, Rev. Mod. Phys. 53, 43 (1981).

[36] J. Preskill, F. Wilczek, and M. Wise, Phys. Lett. 120B, 127 (1983); L. Abbott and P. Sikivie, ibid. 120B, 133 (1983); M. Dine and W. Fischler, ibid. 120B, 137 (1983).

[37] G. Lazarides, C. Panagiotakopoulos, and Q. Shafi, Phys. Lett. B 292, 323 (1987).

[38] T. Banks and M. Dine, Nucl. Phys. B505, 445 (1997).

[39] P. Horava and E. Witten, Nucl. Phys. B460, 506 (1996).

[40] K. Choi, Phys. Rev. D 56, 6588 (1997).

[41] For recent reviews on the different bryogenesis mechanisms, see A. Riotto, hep-ph/9807454; A. Riotto and M. Trodden, Annu. Rev. Nucl. Part. Sci. 49, 35 (1999).

[42] E. W. Kolb and M. S. Turner, Annu. Rev. Nucl. Part. Sci. 33, 645 (1983). 
[43] M. Fukugita and T. Yanagida, Phys. Lett. B 174, 45 (1986).

[44] S. Yu. Khlebnikov and M. E. Shaposhnikov, Nucl. Phys. B308, 885 (1988); J. A. Harvey and M. S. Turner, Phys. Rev. D 42, 3344 (1990).

[45] K. Kumekawa, T. Moroi, and T. Yanagida, Prog. Theor. Phys. 92, 437 (1994).
[46] E. W. Kolb, A. D. Linde, and A. Riotto, Phys. Rev. Lett. 77, 4290 (1996); E. W. Kolb, A. Riotto, and I. I. Tkachev, Phys. Lett. B 423, 348 (1998).

[47] G. F. Giudice, M. Peloso, A. Riotto, and I. I. Tkachev, J. High Energy Phys. 08, 014 (1999). 